\title{
Digitization and astrometric calibration of Carte du Ciel photographic plates with Gaia DR1
}

\author{
K. Lehtinen ${ }^{1}$, T. Prusti ${ }^{2}$, J. de Bruijne ${ }^{2}$, U. Lammers ${ }^{3}$, C. F. Manara ${ }^{2,4}$, J.-U. Ness ${ }^{2}$, H. Siddiqui ${ }^{3}$, T. Markkanen ${ }^{5, \star}$, \\ M. Poutanen ${ }^{1}$, and K. Muinonen ${ }^{1,5}$ \\ ${ }^{1}$ Finnish Geospatial Research Institute, Geodeetinrinne 2, 02430 Masala, Finland \\ e-mail: kimmo.lehtinen@nls.fi \\ 2 Science Support Office, Directorate of Science, European Space Research and Technology Centre (ESA/ESTEC), Keplerlaan 1, \\ 2201AZ Noordwijk, The Netherlands \\ ${ }^{3}$ European Space Astronomy Centre (ESA/ESAC), Camino bajo del Castillo s/n, Urbanización Villafranca del Castillo, \\ Villanueva de la Cañada, 28692 Madrid, Spain \\ ${ }^{4}$ European Southern Observatory (ESO), Karl-Schwarzschild-Straße 2, 85748 Garching bei München, Germany \\ 5 Department of Physics, University of Helsinki, PO Box 64, 00014, Finland
}

Received 18 January 2018 / Accepted 2 May 2018

\begin{abstract}
Context. Carte du Ciel was a global international project at the end of the nineteenth and beginning of the twentieth century to map the sky to about magnitude 14 on photographic plates. The full project was never observationally completed and a large fraction of the observations made remain unanalyzed.

Aims. We want to study whether the astrometric and photometric accuracies obtained for the Carte du Ciel plates digitized with a commercial digital camera are high enough for scientific exploitation of the plates.

Methods. We use a digital camera Canon EOS 5Ds, with a $100 \mathrm{~mm}$ macrolens for digitizing. We analyze six single-exposure plates and four triple-exposure plates from the Helsinki zone of Carte du Ciel $\left(+39^{\circ} \leq \delta \leq+47^{\circ}\right)$. Each plate is digitized using four images, with a significant central area being covered twice for quality control purposes. The astrometric calibration of the digitized images is done with the data from the Tycho-Gaia Astrometric Solution (Gaia TGAS) of the first Gaia data release (Gaia DR1), Tycho-2, Hot Stuff for One Year (HSOY), USNO CCD Astrograph Catalog (UCAC5), and PMA catalogs.

Results. The best astrometric accuracy is obtained with the UCAC5 reference stars. The astrometric accuracy for single-exposure plates is $\sigma(\alpha \cos (\delta))=0.16^{\prime \prime}$ and $\sigma(\delta)=0.15^{\prime \prime}$, expressed as a Gaussian deviation of the astrometric residuals. For triple-exposure plates the astrometric accuracy is $\sigma(\alpha \cos (\delta))=0.12^{\prime \prime}$ and $\sigma(\delta)=0.13^{\prime \prime}$. The $1-\sigma$ uncertainty of photometric calibration is about $0.28 \mathrm{mag}$ and $0.24 \mathrm{mag}$ for single- and triple-exposure plates, respectively. We detect the photographic adjacency (Kostinsky) effect in the triple-exposure plates.

Conclusions. We show that accuracies at least of the level of scanning machines can be achieved with a digital camera, without any corrections for possible distortions caused by our instrumental setup. This method can be used to rapidly and inexpensively digitize and calibrate old photographic plates enabling their scientific exploitation.
\end{abstract}

Key words. astrometry - proper motions - surveys - catalogs - techniques: image processing

\section{Introduction}

Carte du Ciel (hereafter CduC) was a massive international project, initiated in the late nineteenth century, to make photographic charts of the whole sky representing the relative positions of stars down to a photographic magnitude limit of about 14 mag. The $\mathrm{CduC}$ plates proved to be expensive to photograph and reproduce, thus the CduC survey was never fully finished. However, a related photographic survey called Astrographic catalog (or Astrographic Chart, AC), with a lower limiting magnitude of about $11 \mathrm{mag}$, was finished. The scientific motivation of the AC was to determine the proper motions of stars and to study the kinematics of stars in the solar neighborhood. The Observatory of the University of Helsinki completed the AC observations of its zone and measured a total of 284663 star positions, which were published in eight volumes (in 12 books) during the years 1903-1937 (e.g., Donner 1894, 1902; Donner \& Furuhjelm

\footnotetext{
^ Deceased August 28, 2017.
}

1908, 1929). The data of the AC survey have been used for the Tycho-2 catalog (Høg et al. 2000) proper motions by providing an early epoch position, while the more recent position came from the measurements of the HIPPARCOS satellite (ESA 1997) by ESA. The availability of Gaia data makes the CduC plates again interesting as the Gaia proper motions are of such good quality that the Gaia reference frame can be translated beyond a century without much loss of accuracy. Therefore it is possible to establish absolute astrometry for the $\mathrm{CduC}$ plates that is much less limited by the uncertainty of proper motion values than in the case of the Tycho- 2 catalog where accuracy is limited only by precision achievable with the plates.

The optical system in the CduC telescopes is an aplanatic doublet objective, which exhibits some astigmatism and field curvature (Jones 2000). The photographic glass plates are most sensitive to blue light $(\sim 430 \mathrm{~nm})$, with a limiting photographic magnitude of $\sim 14 \mathrm{mag}$. The plates have a total size of $16 \times 16 \mathrm{~cm}$, while the area covered by photographic emulsion is $13 \times 13 \mathrm{~cm}$. To assist in the manual measuring of the star 
positions and to monitor the possible emulsion shifts, the CduC plates have photographically superposed réseau grid lines with $5 \mathrm{~mm}$ separation. The scale of the CduC plates is 1 arcminute per millimetre, and the field of view is $2^{\circ} \times 2^{\circ}$. The plates of our study were taken at the Observatory of the University of Helsinki, around the year 1900 . The declination range of Helsinki covered the range $39^{\circ}-47^{\circ}$ in 1900 equinox. In this paper we carry out a study of a subset of ten plates in order to assess the achievable astrometric accuracy.

The observations in Helsinki were made in a full overlap mode (the corner of a plate is at the center of another plate). The $\mathrm{CduC}$ plates along odd declinations were exposed three times. Each exposure time was $30 \mathrm{~min}$, and the pointing of the telescope was moved by about $10^{\prime \prime}$ between the exposures. Thus, there are three images of each star, forming an equilateral triangle, an asterism. When a star is brighter than $B \approx 10-12 \mathrm{mag}$ the star images in the asterism start to merge together, and consequently an adjacency photographic effect, the so-called Kostinsky effect (Kostinskr 1907; Ross 1921), may arise. The Kostinsky effect increases the relative distances of the stellar images in an asterism as a function of the flux of the star. For CduC triple images, Dick et al. (1993) and Ortiz-Gil et al. (1998) have found the Kostinsky effect, while Geffert et al. (1996) and Bustos Fierro \& Calderón (2003) have not found it.

Previously, the $\mathrm{CduC}$ plates have been digitized with microdensitometers (Dick et al. 1993; Geffert et al. 1996; Rapaport et al. 2006), CCD (Charge Coupled Device) cameras (Bustos Fierro \& Calderón 2003), and flatbed scanners (Vicente et al. 2007, 2010). Microdensitometers are now obsolete (too slow and expensive), while scientific CCD cameras do not have enough pixels to image plates unless one takes a mosaic with a large number of images. Commercial scanners can digitize large plates with good resolution in a reasonably short time, but their problem is a large and non-constant distortion introduced by the scanner itself (Vicente et al. 2007, 2010). However, for a digital camera and lens combination, any distortion is constant, and once determined the distortion can be removed from images.

While digitized Carte du Ciel plates have been previously mainly used for proper motion studies, this is not meaningful in the Gaia era. Our main science goal is to find binary stars with periodicity in the range of several decades to about a century. With Gaia astrometry it is possible to predict precisely where we expect Gaia stars to be on the Carte du Ciel plates at their observing epochs. Most are at the expected positions allowing astrometric absolute calibration of the plates. The science is in the cases where the star is not at the expected position. The astrophysical reason for this can be binarity, which is not resolved by Gaia. For long period unresolved binaries the Gaia proper motion is a combination of the system proper motion and a part of the orbital motion. Thus positional mismatch may be an indication of a binary star system. In addition to this main science goal, the project allows us to explore photometric variability in century timescales and possibly to detect some high ecliptic latitude asteroids.

\section{Method}

To digitize the $\mathrm{CduC}$ plates we used the digital camera Canon EOS 5Ds, which has $8736 \times 5856$ image pixels, together with a Canon EF $100 \mathrm{~mm} \mathrm{f} / 2.8 \mathrm{~L}$ macro IS USM lens. The pixel size is about $11 \mu \mathrm{m}$ corresponding to a nominal resolution of about $0.68^{\prime \prime}$ per pixel. Digital cameras for the consumer market, such as the Canon EOS 5Ds, have a low-pass filter (so called

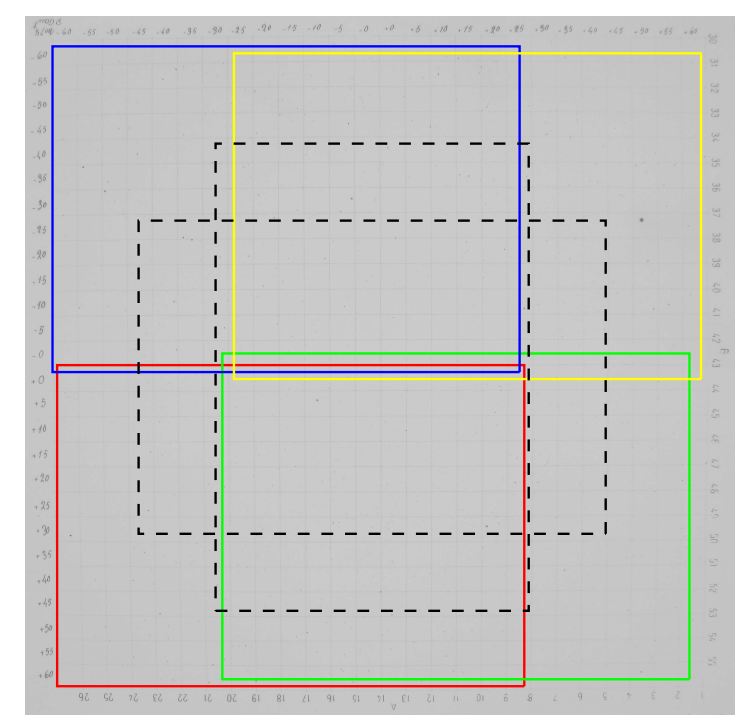

Fig. 1. Schematic view of the two methods used to digitize each plate. The four colored rectangles show the overlapping images that cover the whole plate. The two rectangles with dashed lines show the overlapping images that exclude the borders of the plate. The images taken with the former method are used in our final data analysis.

Table 1. Details of the digitized plates.

\begin{tabular}{lcccc}
\hline \hline Number & $\begin{array}{c}\text { Observing date } \\
(\text { day/mn/year })\end{array}$ & $\begin{array}{c}\text { RA } \\
(1900)\end{array}$ & $\begin{array}{c}\text { Dec } \\
(1900)\end{array}$ & $\begin{array}{c}\text { Expo- } \\
\text { sure }\end{array}$ \\
\hline 841 & $04 / 10 / 1896$ & $20^{\mathrm{h}} 00^{\mathrm{m}}$ & $40^{\circ} 00^{\prime}$ & 1 \\
844 & $06 / 11 / 1902$ & $20^{\mathrm{h}} 00^{\mathrm{m}}$ & $46^{\circ} 00^{\prime}$ & 1 \\
883 & $03 / 10 / 1907$ & $21^{\mathrm{h}} 00^{\mathrm{m}}$ & $40^{\circ} 00^{\prime}$ & 1 \\
886 & $18 / 08 / 1904$ & $21^{\mathrm{h}} 00^{\mathrm{m}}$ & $46^{\circ} 00^{\prime}$ & 1 \\
890 & $27 / 09 / 1904$ & $21^{\mathrm{h}} 10^{\mathrm{m}}$ & $40^{\circ} 00^{\prime}$ & 1 \\
892 & $05 / 11 / 1901$ & $21^{\mathrm{h}} 10^{\mathrm{m}}$ & $44^{\circ} 00^{\prime}$ & 1 \\
854 & $25 / 09 / 1903$ & $20^{\mathrm{h}} 15^{\mathrm{m}}$ & $45^{\circ} 00^{\prime}$ & 3 \\
887 & $13 / 10 / 1896$ & $21^{\mathrm{h}} 05^{\mathrm{m}}$ & $41^{\circ} 00^{\prime}$ & 3 \\
894 & $14 / 10 / 1896$ & $21^{\mathrm{h}} 15^{\mathrm{m}}$ & $41^{\circ} 00^{\prime}$ & 3 \\
896 & $19 / 08 / 1903$ & $21^{\mathrm{h}} 15^{\mathrm{m}}$ & $45^{\circ} 00^{\prime}$ & 3 \\
\hline
\end{tabular}

Notes. The last column states whether the plate is a single- (1) or tripleexposure (3) plate.

anti-alias filter) in front of the sensor. The filter prevents the Moire effect in an image by effectively smoothing an image slightly. In order to have the best possible resolution the filter and the associated optics have been removed by JTW Astronomy $^{1}$ and replaced with an optically polished clear glass filter. This also required a small movement of the detector array to accommodate to the change of the optical path.

Before taking the photos of the CduC plate, the glass is cleaned. The camera is attached to a Kaiser Reproduction Stand RS1/RA1 5510. The glass plates are lit from below by a LED illuminated light table Artograph LightPad A930. To have good enough resolution and to cover the whole plate, we take four partly overlapping images of each plate so that the shorter side of the image sensor covers half the height of a plate $\left(1^{\circ}\right)$ along declination, and the longer side is along right ascension (RA). To check whether the most elongated stellar profiles at the borders of the plates worsen the astrometric accuracy, we digitize

1 http://WwW.jtwastronomy.com 
the plates also by taking two images that are centered at the center of a plate and rotated $90^{\circ}$ with respect to each other. In this case, we exclude the borders of the plates, covering $\sim 50 \%$ of the plate area. These two methods are schematically shown in Fig. 1. In both cases, the central parts of a plate are imaged twice. We disable the corrections for peripheral illumination and chromatic aberration in the camera software. The optical axis of the lens is set perpendicular to the surface of a plate by adjusting the yaw of the camera with the help of the electronic level of the camera. The pitch of the camera is fixed by the stand and cannot be adjusted.

In our project we utilize position and proper motion values of stars given in the Tycho-2 (Høg et al. 2000), USNO CCD Astrograph Catalog (UCAC5; Zacharias et al. 2017), Hot Stuff for One Year (HSOY; Altmann et al. 2017), PMA (Akhmetov et al. 2017) and Tycho-Gaia Astrometric Solution (Gaia TGAS; Michalik et al. 2015) catalogs. The UCAC5, HSOY, and PMA are catalogs between the first data release of Gaia, the Gaia DR1 (Gaia Collaboration 2016a, b; Lindegren et al. 2016), and ground-based astrometry. We limit the UCAC5, HSOY, and PMA catalogs to those stars that have Gaia G-band magnitude less than 16 mag. In Gaia TGAS, the derivation of proper motion and parallax values after only about one year of Gaia observations was made possible by a joint solution of the Tycho-2 catalog with the Gaia DR1 data. The precisions of the proper motions in the Tycho-2, UCAC5, HSOY, and PMA catalogs

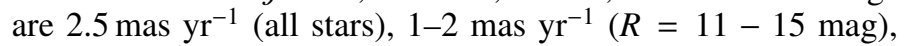

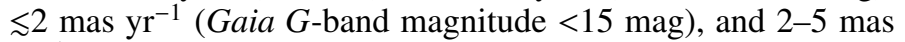
$\mathrm{yr}^{-1}$ (Gaia G-band magnitude 10-17 mag), respectively. For Gaia TGAS data the standard uncertainties are $\sigma\left(\mu_{\alpha \star}\right)=1.1$

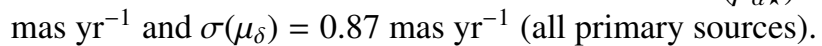

The plates digitized by us are from a region of the sky where the density of the Gaia TGAS stars is at its highest (RA $\approx 19$ h$22 \mathrm{~h}$ ). The typical total number of stars on a CduC plate that can be identified with a star in a reference star catalog is 350 stars with respect to the Gaia TGAS and Tycho- 2 catalogs, and 1713 stars with respect to the UCAC5, HSOY, and PMA catalogs. Table 1 gives details of the digitized plates.

We use the SExtractor program (Bertin \& Arnouts 1996) to search for the stars and to derive their position and flux. SExtractor uses an iterative process to determine the position of a source on an image, and the process works equally well for circular or elliptical objects. We use the SCAMP program (Bertin 2006) to compute the astrometric solutions of the images.

\section{Data analysis}

\subsection{Removal of Bayer pattern}

Images of the CduC plates are saved in a 14-bit RAW format on a hard disk, then converted into a TIFF format using dcraw ${ }^{2}$ program, and then converted into a FITS format for data analysis. A scalar bias value is subtracted from the images. Because the camera has a color sensor, each $2 \times 2$ sub-array of a sensor has one pixel for red, one pixel for blue, and two pixels for green light (Bayer color filter array). We have investigated two ways to remove the Bayer pattern. Firstly, we can use the builtin interpolation options of the dcraw software. In this case, the missing pixels of each color are interpolated, and we get complete images for each of the three colors. The final image is then obtained by taking a weighted mean of the three images. Secondly, we can read the RAW data without any built-in interpo-

\footnotetext{
2 http://www. cybercom.net/ dcoffin/dcraw
}

lation within dcraw. Then, the mean values of the red and the blue pixels are scaled to the mean value of the green pixels over the whole image. These two methods give practically the same results for the astrometry, but we have used the latter method for our images. In this method, the scaling factors are determined mostly by the background pixels, which form the vast majority of an image. This method works as long as the spectrum of the light coming through a plate stays the same. Some plates have yellowish regions at their borders where scaling residuals can be seen at the $\sim 5 \%$ level. Furthermore, the plateaus at the centers of some stars show scaling residuals at the $\sim 1 \%$ level. The final astrometric accuracy is not sensitive to the accuracy of the scaling correction; changing the scaling factors for any color by $\pm 10 \%$ increases the residuals of the astrometry only by about $0.02^{\prime \prime}$.

\subsection{Removal of grid lines}

When searching for stars in an image with SExtractor, the image is first heavily smoothed to derive the background value at each pixel. Before smoothing, the grid lines have to be removed. Our procedure is the following. Suppose we want to remove the lines running along the $y$-axis. We take a mean value over each column, obtaining a vector giving the shapes of the grid lines with a very good signal-to-noise ratio as a function of the $x$-axis of an image. Finding the positions of the grid lines is then finding the positions where the derivative of the vector changes from large positive values to large negative values. This is a robust method because it does not depend on the absolute value of the intensity. We then linearly interpolate the pixels which are \pm seven pixels around the center of the grid line, and the interpolated pixels are flagged as bad pixels. The flagged pixels constitute about $7 \%$ of the total image area. All the stars that have a bad pixel within their stellar profile are discarded.

\subsection{Astrometric solution}

The astrometric and photometric analysis of each plate consists of the following steps:

(i) Preliminary astrometry for the image is obtained with the Astrometry.net software (Lang et al. 2010). The purpose of this stage is to get a preliminary astrometry that SCAMP software can successfully use as a starting point in the refinement of the astrometry (step iv below).

(ii) the SExtractor software is used to detect the objects in the image and to derive their fluxes and pixel positions.

(iii) The coordinates of the stars in the external catalogs are transformed to the epoch of the plate to be used as reference stars for astrometry. The transformation is made as described in Sect. 1.5.5 of Volume 1 of ESA (1997). Also the uncertainties of the transformed coordinates are computed.

(iv) The SCAMP software is used to compute astrometric projection parameters by using the pixel positions of stars from step ii and the reference stars from step iii above, after dividing each image into two sub-images along the shorter side of the image array. SCAMP derives a polynomial distortion model for an image by minimizing a weighted quadratic sum of the differences in the coordinates (longitude and latitude) between the stars in the plate and the reference stars. A distortion of an image can be visualized as a variation of the pixel scale as a function of coordinates. The astrometric fit by SCAMP in the sub-images is computed by using an average of 78 reference stars in the case of the Gaia TGAS and Tycho- 2 catalogs, and an average of 181 stars in the case of 
the UCAC5, HSOY, and PMA catalogs. Due to the relatively low number of reference stars in our sub-images, we use a low, second-order polynomial in the astrometric fit as a starting point. Then, based on visual examination of check-plots produced by SCAMP and the value of $\chi^{2}$ of the astrometric fit, we use a third-order polynomial if necessary. In the case of a second-order polynomial, the equation for the longitudinal distortion has the form

$x^{\prime}=a+b x+c y+d x^{2}+e x y+f y^{2}$,

where $x^{\prime}$ is the distortion-corrected longitude, $x$ and $y$ are uncorrected longitudinal and latitudinal offsets from the distortion-center origin, and $a$ to $f$ are constants. A similar equation applies for the latitude, by interchanging $x$ and $y$ (Shupe et al. 2005).

(v) The distortion parameters computed by SCAMP are fed to the header of the image and SExtractor is run again to derive the final celestial coordinates of the objects at the epoch of each plate.

\subsection{Triple exposures}

We use SExtractor to detect the star images in the triple exposures. The location of each star image relative to the two closest star images is used to deduce which star images are members of a certain triplet. In this way, we detect such triplets where the individual star images are detected as distinct objects by SExtractor. The star images with a visual magnitude $\lesssim 8$ mag remain undetected because their triplets cannot be deblended into three distinct objects. However, the number of such star images is small.

We fit the triplets with three overlapping elliptical Gaussians with a saturation parameter (Dick et al. 1993). The width of all the three Gaussians is expected to be equal. The fitted function for a triplet has the form

$$
\begin{aligned}
I_{i, j}= & B+\sum_{k=1}^{3} A_{k} \exp \left\{-\frac{1}{2}\left[\frac { 1 } { 1 - t ^ { 2 } } \left(\left(\frac{x_{i j}-x_{c k}}{\sigma_{x}}\right)^{2}\right.\right.\right. \\
& \left.\left.\left.+\left(\frac{y_{i j}-y_{c k}}{\sigma_{y}}\right)^{2}-2 t\left(\frac{x_{i j}-x_{c k}}{\sigma_{x}}\right)\left(\frac{y_{i j}-y_{c k}}{\sigma_{y}}\right)\right)\right]^{s}\right\},
\end{aligned}
$$

where $B$ is the value of the background, $x_{i j}$ and $y_{i j}$ are the pixel coordinates, $x_{c k}$ and $y_{c k}$ are the center coordinates of the $k$ th Gaussian function, $A_{k}$ is the peak intensity of the Gaussian function, $\sigma_{x}, \sigma_{y}$ and $t$ are widths and orientation of the elliptical Gaussian functions, and $s$ is the flattening (saturation) parameter. The center position of the triplet in the pixel coordinates, $(x, y)$, is determined as a mean value of the $x$ - and $y$-positions of the three Gaussians. Then, we follow the analysis steps given in Sect. 3.3 above, excluding step ii.

\subsection{Removal of instrumental distortions}

The distortions caused by our instrumental setup include the distortions by the camera plus lens combination, and the distortions caused by the non-perpendicularity of the optical axis of the lens relative to the plate. Our procedure for removing these distortions is the following:

(i) Make a fake, random stellar field and print it on a paper. Also, make a corresponding star catalog in a format that SCAMP can read.

(ii) Take several images of the field in a dither pattern, that is, move the paper slightly between the exposures.

(iii) Use SExtractor to derive the positions of the artificial stars.

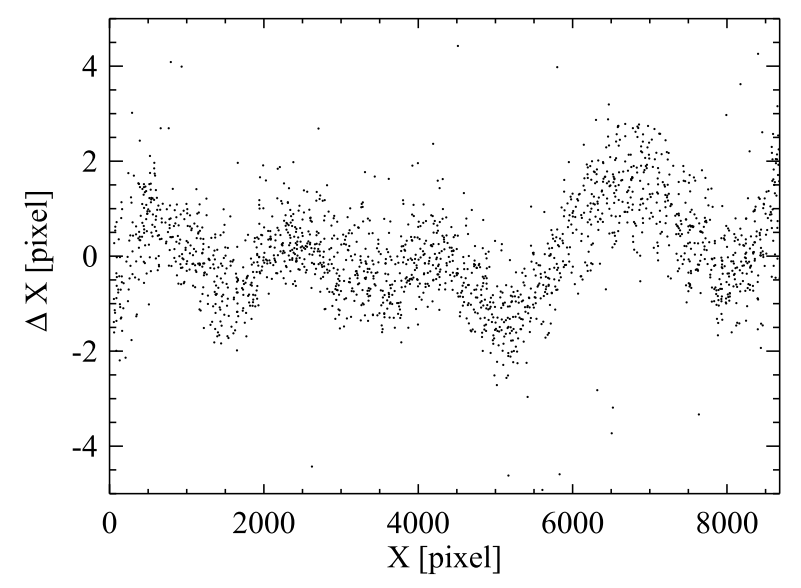

Fig. 2. Difference, along the $x$-axis of an image, between the modeled and true of a paper and/or a drum in a printer.

(iv) Use SCAMP to derive the distortions for each image, based on the positions derived in the previous step, and take a mean value of the distortion parameters.

(v) Use the SWarp program (Bertin et al. 2002) to correct the $\mathrm{CduC}$ images for the instrumental distortions.

Currently, our procedure for determining and correcting the distortions caused by our instrumental setup (Sect. 3.5) is hampered by two facts:

(i) Consumer-grade laser printers suffer from an uneven movement of the drum and/or paper at a level that is significant for us. This is illustrated in Fig. 2, which shows the one-dimensional difference, along the $x$-axis of an image, between the modeled and true coordinates of the artificial reference stars printed on a paper. The $x$-axis is along the movement of a paper inside the printer. The wavy relation is not seen along the $y$-axis, perpendicular to the movement of a paper.

(ii) A proper determination of the distortion requires an average over several distortion maps of an artificial stellar field. However, we have found no way to derive an average of the distortion parameters when processing simultaneously several images of the artificial stellar field using SCAMP. Due to the above-mentioned facts, a correction for the instrumental distortion is not implemented into our current data analysis. With our method the instrumental distortion is simply removed as part of astrometric distortion done separately for each plate.

\subsection{Photometric calibration}

The spectral response of our plates is not known, but the response peaks at blue wavelengths. Therefore, we make photometric calibration for each plate separately by forming a relation between the logarithm of the total flux of a star on a CduC plate and its Tycho-2 $B_{T}$ magnitude.

\section{Results}

The histograms of uncertainty of RA $(\sigma(\alpha \cos (\delta)))$ and declination $(\sigma(\delta))$ of the Gaia TGAS, UCAC5, Tycho-2, HSOY, and PMA reference stars within the areas of our plates, after transforming their coordinates into the epoch of each plate, are shown in Fig. 3. The error analysis includes the uncertainties of the coordinates (at the epoch of each catalog), the uncertainties of the 


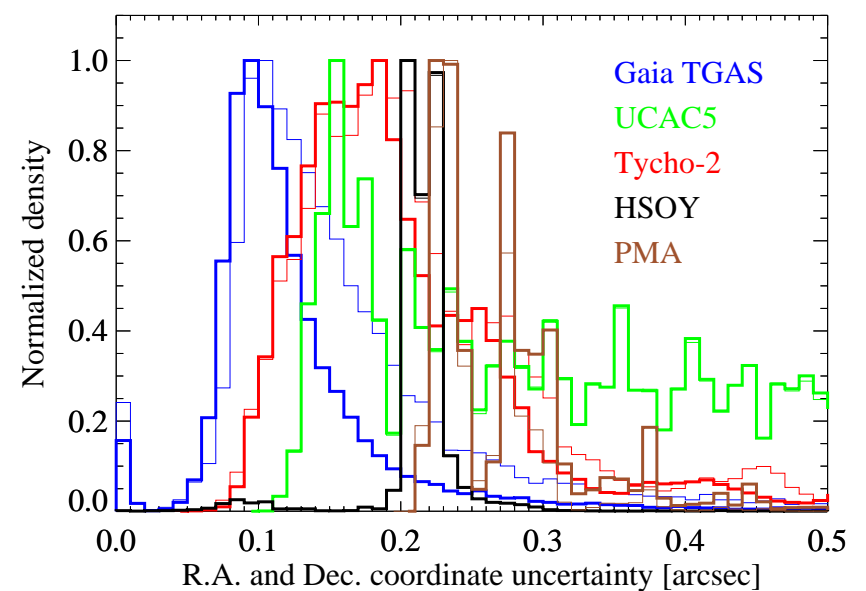

Fig. 3. Histograms of uncertainty of RA and declination of the Gaia TGAS (blue), UCAC5 (green), Tycho-2 (red), HSOY (black), and PMA (brown) reference stars, after transforming their coordinates into the epochs of our plates. The thin line is for RA, the thick line is for declination. Only the stars within the areas of our plates are included.

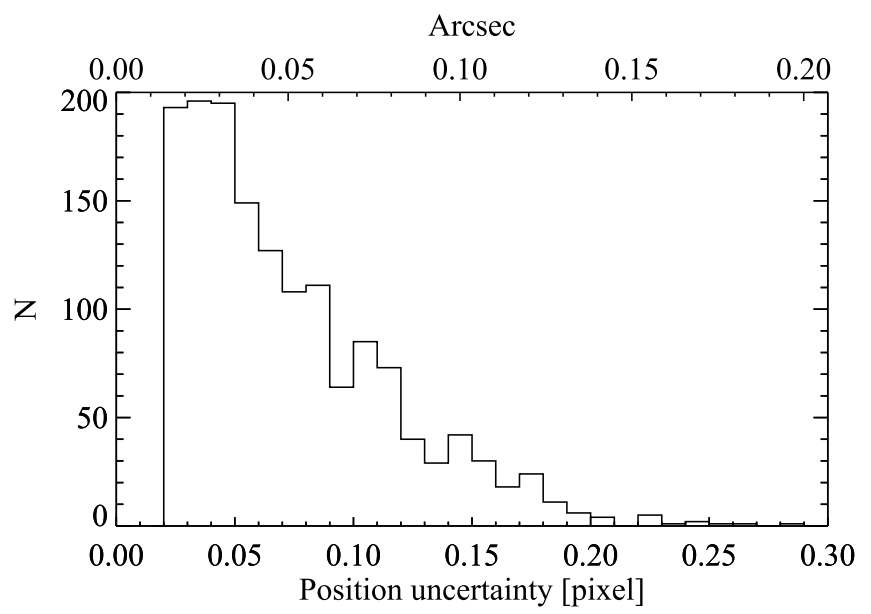

Fig. 4. Example of a histogram of standard deviations of positions for the stars on one of our single-exposure image, as measured by SExtractor. The histogram is a mean value of the deviations along the $x$ - and $y$-axis of an image.

proper motion values, and, for Gaia TGAS data, also the correlations between the observational parameters. Both for the Gaia TGAS and Tycho- 2 data, the uncertainty is greater along the RA due to the scanning strategy of the satellites. To check whether the obtained astrometric accuracy is limited by the proper motion uncertainties of the reference stars, we compute the astrometric solutions also when using only those Gaia TGAS, UCAC5, HSOY, and PMA reference stars which have uncertainties of the coordinates less than $0.15^{\prime \prime}, 0.20^{\prime \prime}, 0.27^{\prime \prime}$, and $0.32^{\prime \prime}$ at the epoch of each plate, respectively.

Figure 4 gives an example of the standard deviations of the pixel positions of stars on an image, as computed by SExtractor. The deviations are mean values of the (very similar) deviations along the $x$-and $y$-axis of an image. Figure 5 shows examples of the stellar profiles of a star at the corner and center of a CduC plate, with Gaussian Full Width at Half Maximum (FWHM) sizes of $\sim 6^{\prime \prime} \times 10^{\prime \prime}$ and $\sim 7^{\prime \prime}$, respectively. Thus, within the stellar profiles there may be dimmer, unseen stars, which move the photo-center of the star seen on a plate away from its expected position. This effect is more serious at the corners of the plates because the stellar profiles are larger there.
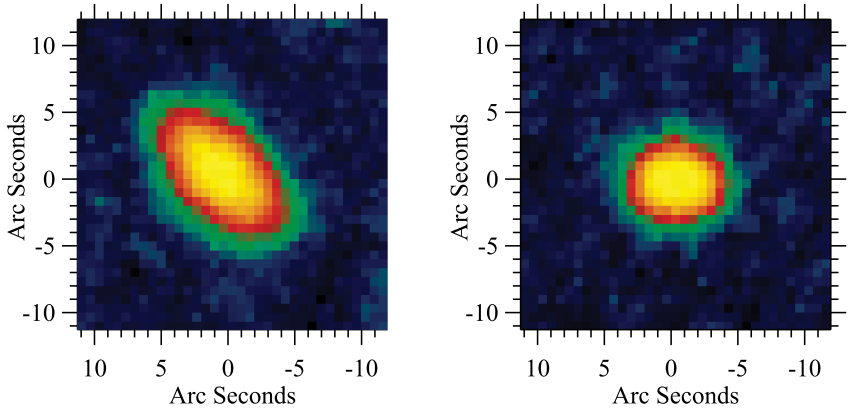

Fig. 5. Examples of stellar profiles on our images. The left and right panels show profiles at a corner and center of a plate, respectively. The Tycho- $2 B_{T}$ magnitudes of the stars are 12.8 (left panel) and 12.5 (right panel).

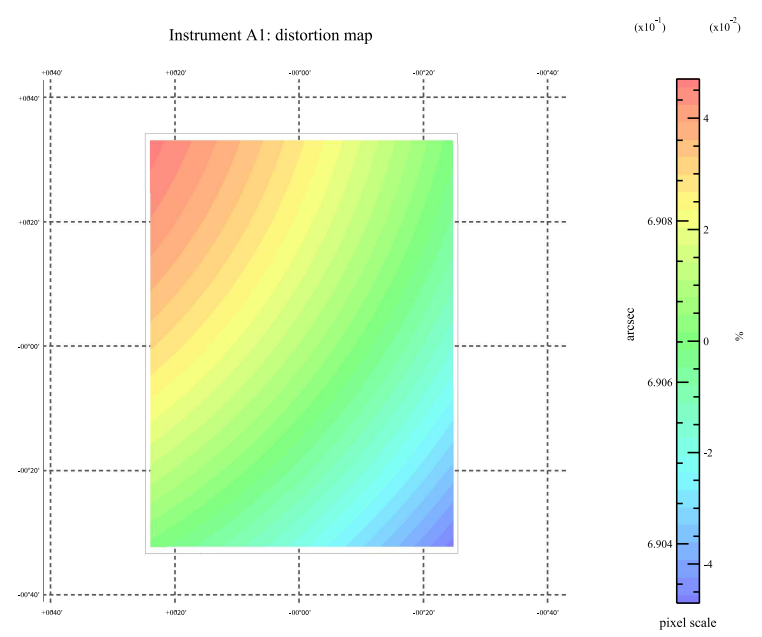

Fig. 6. Example of a distortion map produced by an astrometric fit when using Gaia TGAS reference stars. The map is for one of the sub-images of the plate \#892. The color bar gives the pixel scale of the image in absolute and percentual scales. The plot was produced by SCAMP.

\subsection{Astrometric distortion}

An example of a distortion map is shown in Fig. 6. Values of distortions over an image vary from about \pm 0.02 to $\pm 0.4 \%$. We expect that the distortion map of the refractor itself used to image the plates has the largest pixel scale coinciding with the optical axis, that is, at the center of a plate. While some of our images do show such a distortion map, as the one shown in Fig. 6, we believe that in most cases the distortion maps are dominated by distortions caused by the non-perpendicular orientation of the optical axis of the camera relative to the plate.

For plates \#841 and \#890, a second-order polynomial cannot properly fit the astrometric distortions. This is depicted, for example, by a skewed distribution of the difference between the fitted and cataloged coordinates of the reference stars, at the epoch of a plate. Using a third-order polynomial produces a nonskewed distribution. By visually checking the distortion map produced by a third-order polynomial fit we confirm that distortion changes over scales that are larger than the typical distance between the reference stars, thus distortion is not over-fitted.

\subsection{Astrometric analysis}

Our astrometric analysis includes the following steps; (i) Computing astrometry for quality checking of each plate, 


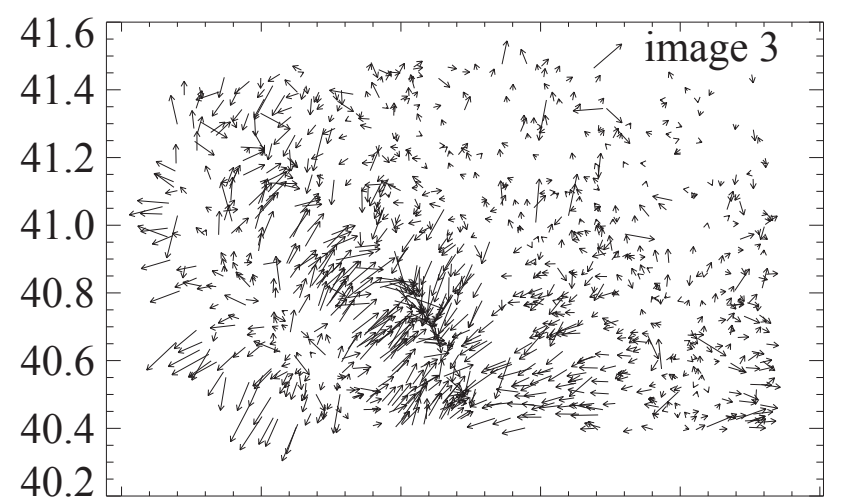

$\begin{array}{llllll}320.0 & 319.5 & 319.0 & 318.5 & 318.0 & 317.5\end{array}$
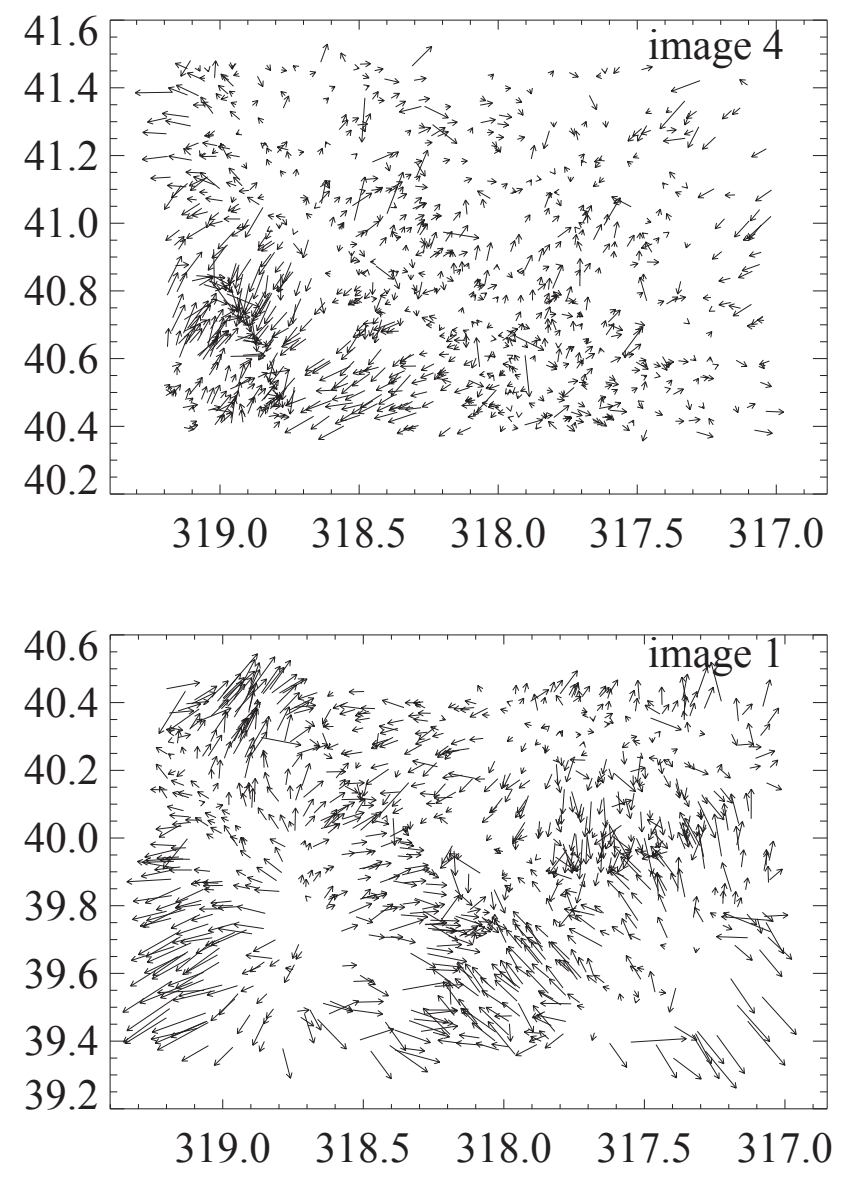

Fig. 7. Large-scale astrometric vector residuals. For each UCAC5 reference star which is used to fit the astrometry of an image, the arrow gives the magnitude and direction of the difference between the coordinates of the star, at the epoch of the plate, and the fitted coordinates of the corresponding star on the CduC image. The data is shown separately for each of the four partly overlapping images of plate \#890. The scale is shown in the lower right corner of image 2.

(ii) Computing astrometry for each plate that is of good quality and correcting the stellar coordinates for possible biases, and (iii) evaluating the astrometric fits. These steps are discussed in the next three sections. Whenever we correlate the source coordinates on two lists we use a search radius of $2^{\prime \prime}$.

\subsubsection{Astrometric fit for quality checking}

The results in this section are given in the case of covering the plates with four overlapping images (see Fig. 1), and by simultaneously processing all the partly overlapping eight sub-images of each plate with SCAMP. The accuracy of the astrometric solutions for the plates are given in Table A.1 as Root Mean Square (RMS) deviations of the astrometric fits along the axes of the images (dAXIS1 and dAXIS2 in the notation by SCAMP, along right ascension and declination in our case, respectively). The internal calibration represents the field-to-field calibration of the overlapping fields, while the external calibration represents the calibration relative to the reference stars. For the Gaia TGAS, UCAC5, HSOY, and PMA data, the results are given both for the case of using all the stars, and using only those stars for which the coordinate uncertainties, at the epoch of the plate, are less than the limit given. In the latter case, the accuracy of astrometry is marginally improved for the Gaia TGAS and UCAC5 data. However, the improvement in astrometry is only about $0.02-0.03^{\prime \prime}$, and the number of the reference stars is further reduced. Therefore all the further data analysis is done only for the case of using all the reference stars in the external catalogs.

As discussed in Sect. 2 and shown in Fig. 1, the plates have been digitized also by taking two overlapping images, centered at the center of the plate, and excluding the borders of the plate. In that case the mean values of dAXIS1 and dAXIS2 for the single-exposure plates, when using UCAC5 reference stars, are $0.17^{\prime \prime}$ and $0.16^{\prime \prime}$. These values are to be compared with the corresponding values in Table A.1, $0.21^{\prime \prime}$ and $0.20^{\prime \prime}$. Thus, the accuracy of astrometry is improved by $\sim 0.04^{\prime \prime}$ when excluding the borders of the plates.

Table A. 1 shows that the deviation of the astrometric solution differs between the plates by a factor of about two. Particularly, the values of dAXIS and $\chi^{2}$ for plate \#890 are clearly larger than the values for the other plates. Visual inspection of the images shows no difference in the quality between these plates. Furthermore, the uncertainties of the pixel positions of stars are similar for all the plates. To check whether the astrometric residuals show structure on a large scale, we make an astrometric fit for each full image (not dividing the image into two sub-images), using a second-order polynomial in the fit, and plot the astrometric vector residuals. Figure 7 shows the astrometric residuals 


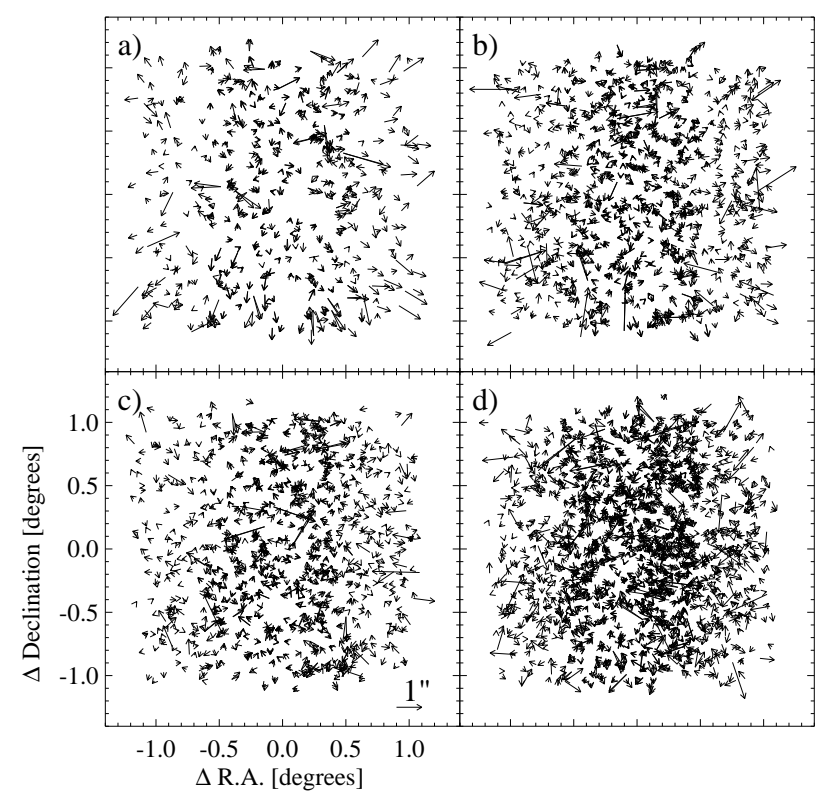

Fig. 8. Vector residuals for the UCAC5 reference stars in the singleexposure plates, within four different magnitude ranges. The Gaia $G$-band magnitudes of the ranges are $<10 \mathrm{mag}$ (panel a), 11.0-11.5 mag (panel b), 12.0-12.25 mag (panel c), and 13.0-13.25 mag (panel d).

for each image of plate \#890. There are regions where the direction and magnitude of the residuals changes abruptly, such as in the north-west corner of image 2 . The vectors are not oriented in a way that is expected for a magnitude equation (pointing radially outwards from the center of the plate), except at the southwest corner of image 1 . We conclude that the intrinsic quality of plate \#890 is lower than that of the other plates, possibly due to a large-scale deterioration of the emulsion. Plate \#841 shows a similar behavior, but with smaller values of residuals. Plate \#890 is included in the results of Table A.1, but further data analysis is done without plate \#890.

\subsubsection{Biases in the stellar coordinates}

The results in this section are given in the case of covering the plates with four overlapping images (see Fig. 1), and after individually processing all the partly overlapping eight subimages of each plate with SCAMP. It is known that the photographic plate material may suffer from a magnitude-dependent systematic bias in the positions of the stars, the so-called magnitude equation (e.g., Girard et al. 1998; Vicente et al. 2010). It is caused by the combination of asymmetric stellar profiles and the nonlinear response of photographic emulsions. As a result, the photographic density profiles of stars are skewed, so that the profiles of stars are more skewed for the brighter stars, which are more affected by the nonlinearity of the emulsion. The asymmetry itself can be caused by, for example, optical aberrations or tracking errors during an exposure. We believe that for the CduC plates the asymmetry is dominated by field curvature, which produces elongated stellar profiles with the long axis of the profile pointing towards the center of the plate (Ortiz-Gil et al. 1998) (see Fig. 5).

The differences between the fitted and cataloged coordinates (vector residuals) of the UCAC5 stars in the single-exposure plates, within different magnitude ranges, are shown in Fig. 8. The magnitude of the residuals is largest for the brightest stars, as expected for magnitude equation. The residuals are pointed outward from the center of the plate, similarly to the magnitude

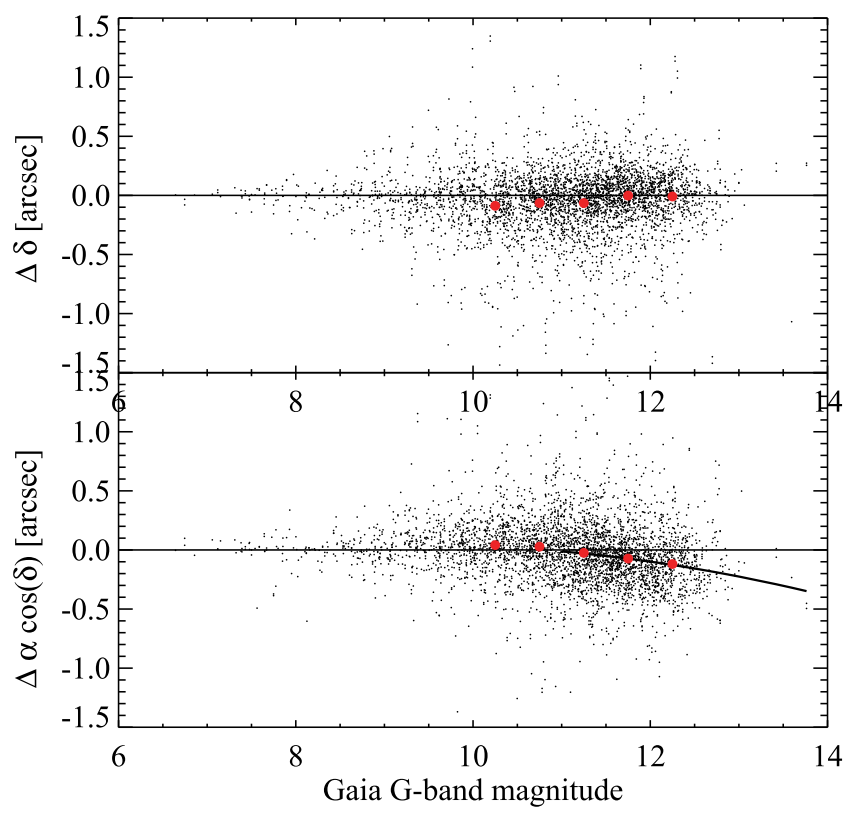

Fig. 9. Difference between the fitted and cataloged coordinates of all the Gaia TGAS stars in our single-exposure plates as a function of the Gaia $G$-band magnitude. Mean values over 0.5 magnitude bins are shown as red dots. The lower panel is for RA, the upper panel is for declination. Overplotted in the lower panel is a second-order polynomial fit for magnitudes $>11$ mag.

equation found by Vicente et al. (2010) for CduC plates. However, the number of stars is too low to determine an accurate correction for magnitude equation in our plates.

Magnitude equation is barely visible with the Gaia TGAS reference stars. It is possible that due to the lower number of Gaia TGAS reference stars, the SCAMP is able to fit some of the distortion caused by magnitude equation. Related to the magnitude equation, the distances between all the stars detected in the single-exposure plates and their counterparts in the Gaia TGAS catalog (i.e., astrometric residuals) as a function of the Gaia $G$-band magnitude are plotted in Fig. 9. At magnitudes $\gtrsim 11$ the fitted RA coordinates of stars start to deviate from their cataloged coordinates. Overplotted in the lower panel of Fig. 9 is a second-order polynomial fit, which is subtracted from the fitted RA coordinates of those stars that have Gaia G-band magnitude greater than $11 \mathrm{mag}$. The corresponding image for the tripleexposure plates is in Fig. 10. There is a similar bias in the RA coordinates than for the single-exposure plates, but the bias is not clear enough to be fitted and subtracted.

The astrometric residuals for the Tycho-2 stars as a function of the Gaia $G$-band magnitude are plotted in Fig. 11. There is a small bias in the residuals along declination, with a maximum value of about $-0.07^{\prime \prime}$. We do not correct this bias. The astrometric residuals for the UCAC5 stars in the single-exposure plates as a function of the Gaia $G$-band magnitude are plotted in Fig. 12. There is no bias in the residuals. The same is true for the triple-exposure plates. The astrometric residuals for the HSOY stars in the single-exposure plates as a function of the Gaia $G$-band magnitude are plotted in Fig. 13. The residuals along declination are biased, and overplotted in the upper panel is a second-order polynomial fit, which is subtracted from the fitted declination coordinates of those stars that have Gaia $G$-band magnitude greater than $12 \mathrm{mag}$. The residuals along RA show a small bias but we do not correct it. There is no clear bias in a similar plot for the triple-exposure plates. The astrometric 


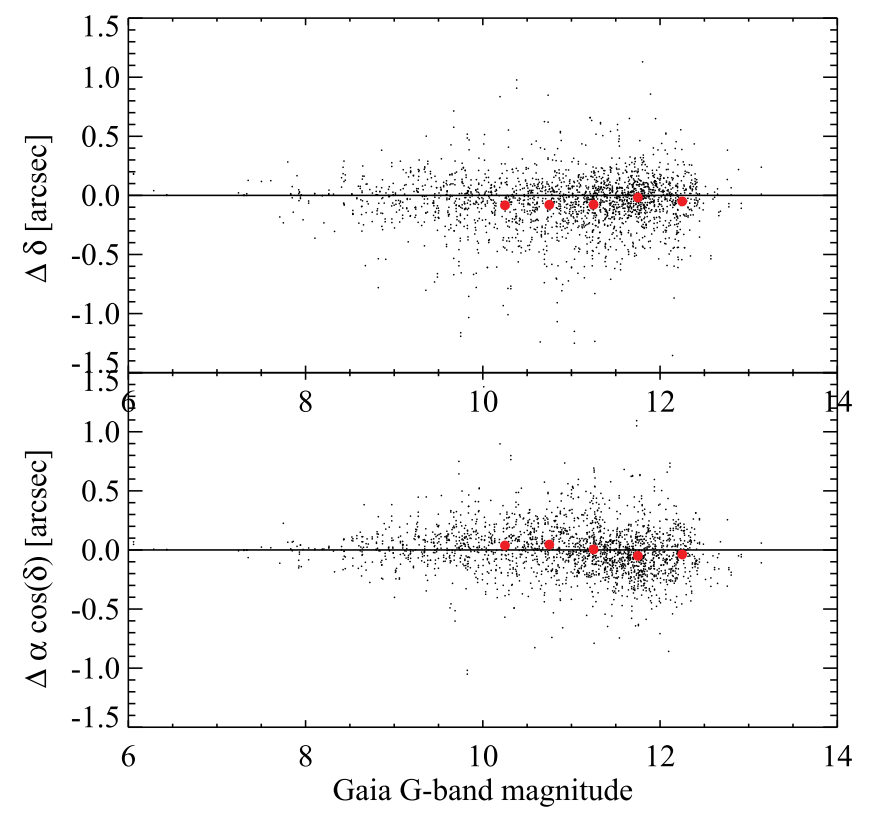

Fig. 10. Difference between the fitted and cataloged coordinates of all the Gaia TGAS stars in our triple-exposure plates as a function of the Gaia $G$-band magnitude. Mean values over 0.5 magnitude bins are shown as red dots. The lower panel is for RA, the upper panel is for declination.

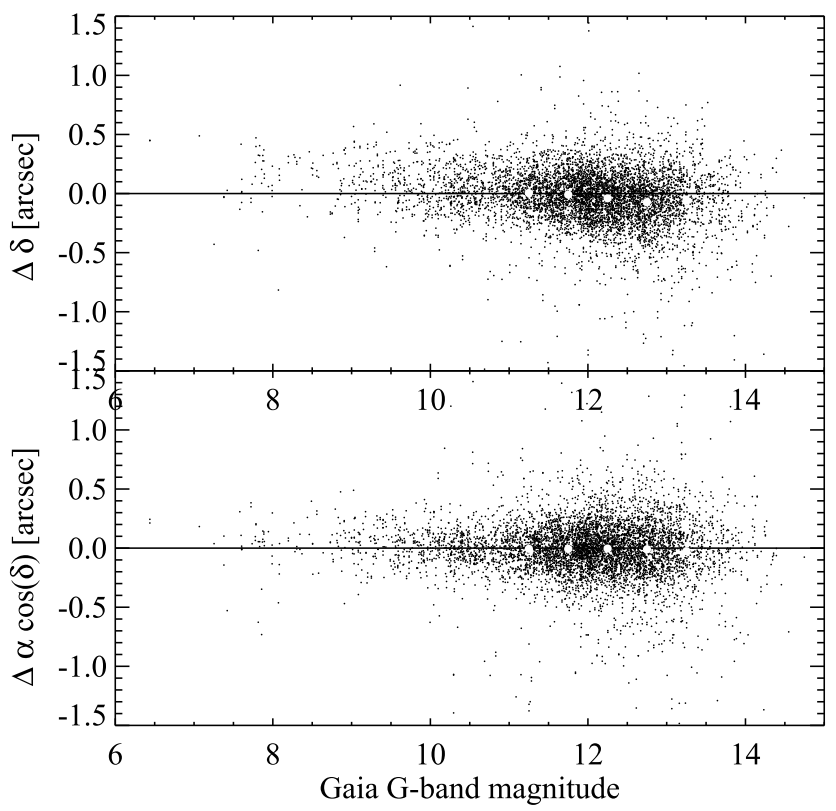

Fig. 11. Difference between the fitted and cataloged coordinates of all the Tycho-2 stars in our plates (both single- and triple-exposure) as a function of the Gaia $G$-band magnitude. Mean values over 0.5 magnitude bins are shown as white dots. The lower panel is for RA, the upper panel is for declination.

residuals for the PMA stars in the single-exposure plates as a function of the Gaia G-band magnitude are plotted in Fig. 14. There is no bias in the residuals. The same is true for the tripleexposure plates.

We conclude that the coordinates of the Gaia TGAS and HSOY reference stars, at the epoch of the plates, are biased along RA and declination, respectively, as a function of magnitude.

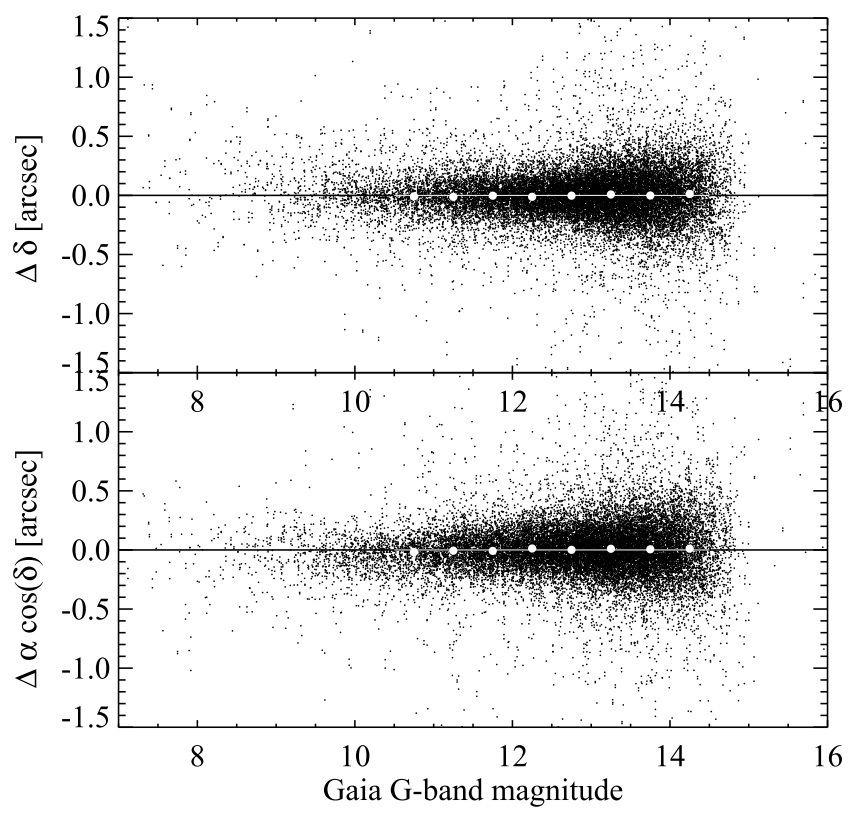

Fig. 12. Difference between the fitted and cataloged coordinates of all the UCAC5 stars in our single-exposure plates as a function of the Gaia $G$-band magnitude. Mean values over 0.5 magnitude bins are shown as white dots. The lower panel is for RA, the upper panel is for declination.

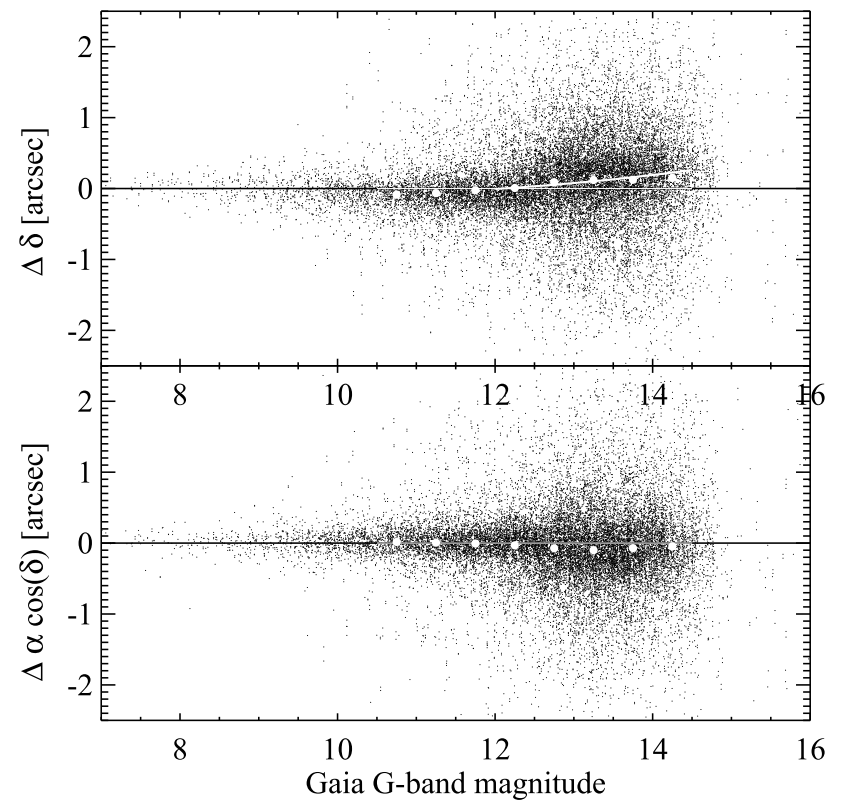

Fig. 13. Difference between the fitted and cataloged coordinates of all the HSOY stars in our single-exposure plates as a function of the Gaia $G$-band magnitude. Mean values over 0.5 magnitude bins are shown as white dots. The lower panel is for RA, the upper panel is for declination. Overplotted in the upper panel is a second-order polynomial fit for magnitudes $>12$ mag.

We also check whether the difference between the fitted and cataloged coordinates of the Gaia TGAS stars in our plates is correlated with the properties of those stars (other than the correlation in Fig. 9). The properties include ellipticity of a star, position angle of an elliptic stellar profile, width of a stellar profile, pixel position of a star on a plate, and color of a star (Tycho-2 $B_{T}$ and $V_{T}$ magnitudes). We find no correlation. The differences between the coordinates of the Tycho-2 and Gaia 


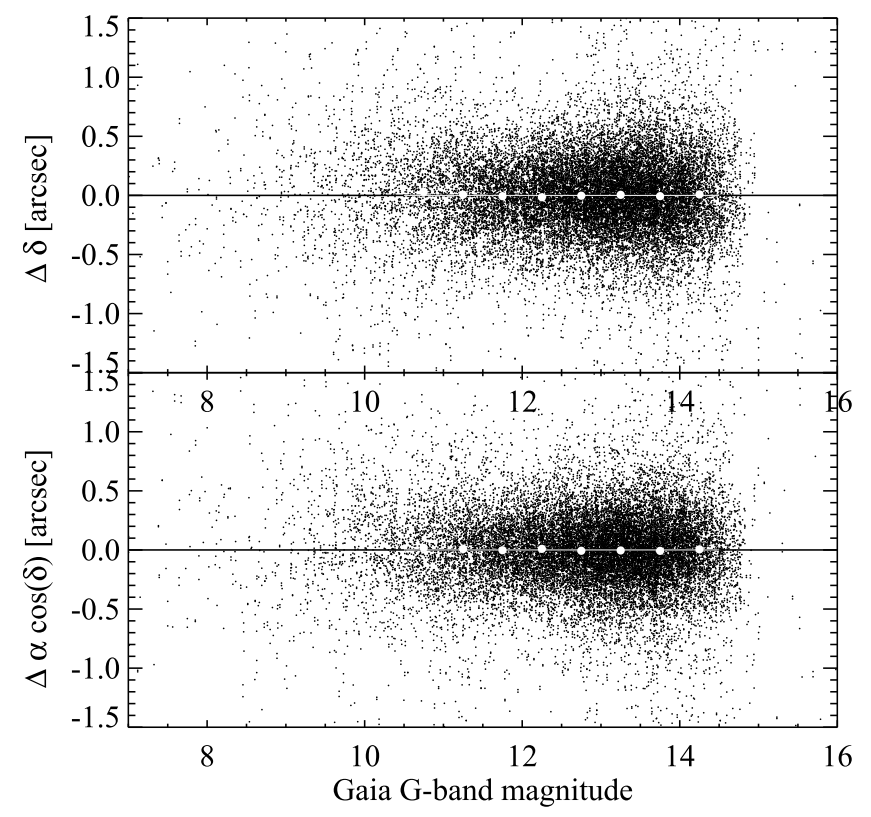

Fig. 14. Difference between the fitted and cataloged coordinates of all the PMA stars in our single-exposure plates as a function of the Gaia $G$-band magnitude. Mean values over 0.5 magnitude bins are shown as white dots. The lower panel is for RA, the upper panel is for declination.

TGAS stars, at the epoch of the plates, do not show any correlation with the properties of the corresponding stars on a $\mathrm{CduC}$ plate.

Figure 15 shows the differences between the Gaia TGAS and UCAC5 proper motions for the stars within our plates, as a function of Gaia $G$-band magnitude. There is a bias in RA proper

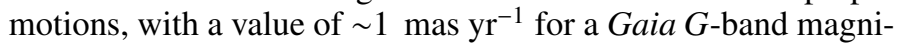
tude of $\sim 12$. Within a time span of 100 years, this corresponds to a RA coordinate bias of $\sim 0.1^{\prime \prime}$, close to the value that we observe with Gaia TGAS reference stars in Fig. 9. We conclude that the bias in the astrometric residuals of the Gaia TGAS stars along RA is probably caused by a bias in the values of the RA proper motions in the Gaia TGAS catalog. An equivalent plot of the proper motion differences between the HSOY and UCAC5 stars as a function of Gaia $G$-band magnitude shows a bias along declination, which could explain the bias seen in Fig. 13.

\subsubsection{Astrometric accuracy}

The astrometric accuracy of the final stellar coordinates can be evaluated in three ways: (i) the deviation between the coordinates of a star in two overlapping images of the same plate, (ii) the deviation of the coordinates between all the stars detected and their counterpart in the reference star catalog, at the epoch of each plate, and (iii) the deviation of the stellar coordinates in the overlapping plates.

(i) The differences between the fitted coordinates of the UCAC5 stars in the overlapping images of the single- and tripleexposure plates are plotted in Figs. 16a and 17a, respectively. The Gaussian standard deviations of the differences are given in Table 3.

(ii) The differences between the fitted coordinates of all the stars detected and their counterparts in the Tycho-2, Gaia TGAS, and UCAC5 catalogs are plotted in Figs. $16 \mathrm{~b}-\mathrm{d}$ and $17 \mathrm{~b}-\mathrm{d}$, for the single- and triple-exposure plates, respectively. The Gaussian standard deviations of the differences are given in the Table 3.
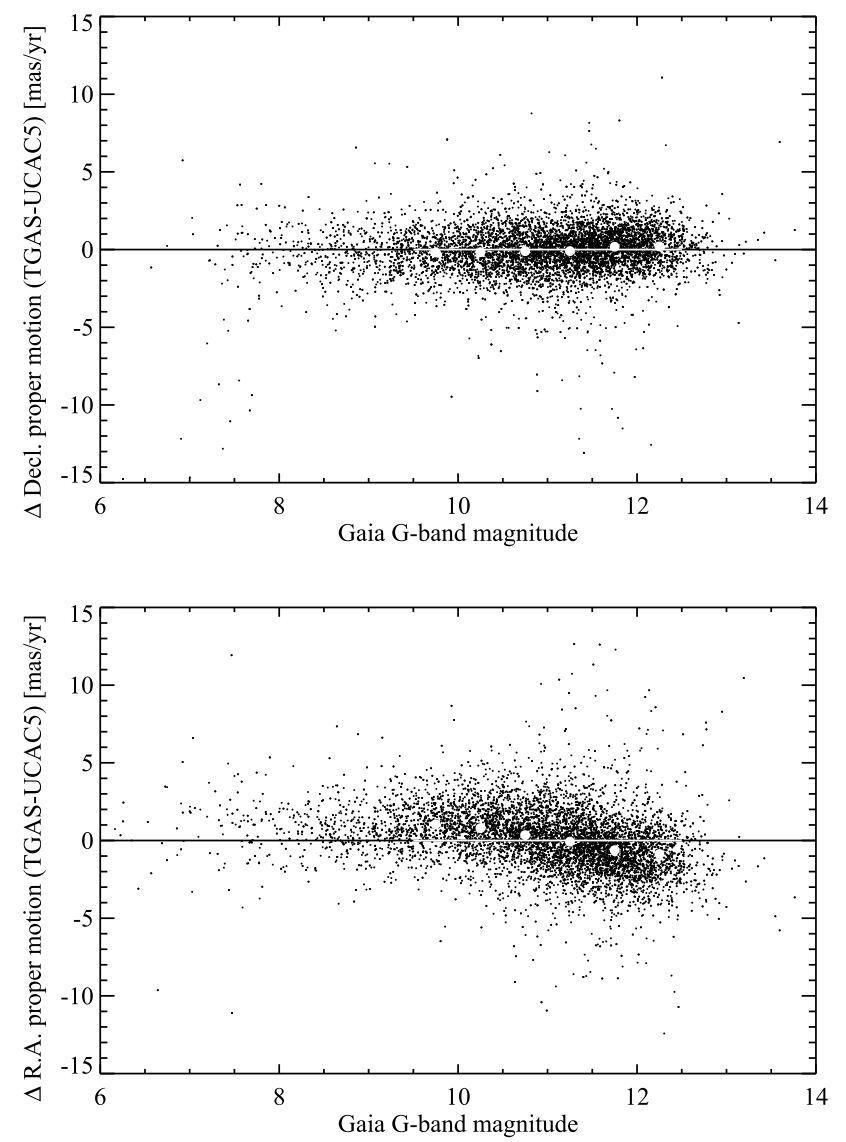

Fig. 15. Differences of the proper motions given in the Gaia TGAS and UCAC5 catalogs as a function of magnitude for the stars within our plates. Mean values over 0.5 magnitude bins are shown as white dots. The lower panel is for RA, the upper panel is for declination.

(iii) The differences of the fitted coordinates of the stars which are common to the partly overlapping plates \#892 and \#896 are shown in Fig. 18.

The pixel positions of stars measured by SExtractor are Gaussian-weighted centroids of stellar profiles. To check whether the accuracy of astrometry depends on the method to determine the pixel positions of the stars, we perform our analysis for some single-exposure plates also by fitting the stellar profiles with a two-dimensional (2D) Gaussian function. The astrometric accuracy is then practically the same as in the case of using SExtractor.

\subsection{Photometry}

Figure 19 shows an example of a relation between the total flux of stars, measured from a CduC plate, and the Tycho- $2 B_{T}$ magnitudes. A second-order polynomial is fitted to the relation and residuals are formed by subtracting the fit from the $\mathrm{CduC}$ based magnitudes. The corresponding residuals for all the stars in the single-exposure plates are shown in Fig. 20 as a function of the distance of the star from the center of the plate. Near the center of the plate the stars are dimmer, while near the edges of the plate the stars are brighter than expected. The trend in Fig. 20 is fitted with a straight line, the line is subtracted from the residuals, and the standard deviation of the residuals, $\sim 0.28 \mathrm{mag}$, is used as a measure of photometric accuracy for the $\mathrm{CduC}$ stars. There is no correlation between the magnitude residuals and the colors (Tycho-2 $B_{T}$ minus $V_{T}$ magnitudes) or the Tycho-2 $B_{T}$ magnitude of the stars. The corresponding 

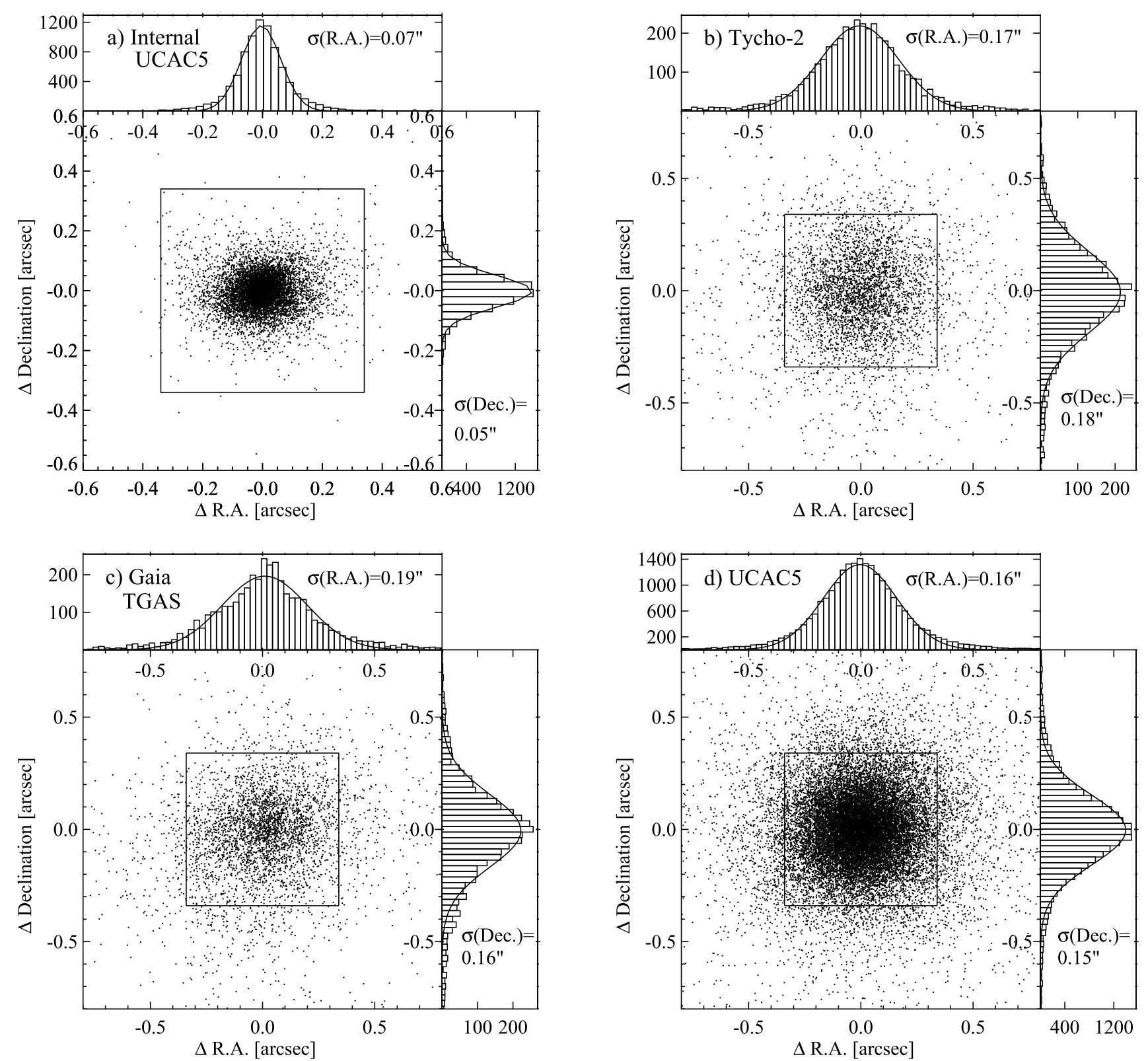

Fig. 16. Astrometric results for the single-exposure plates. Panel $a$ : the differences between the coordinates of the UCAC5 stars in the overlapping images at the epoch of each plate. Panels $b-d$ : the differences between the coordinates of all the stars in the plates and the coordinates of the corresponding stars in the Tycho-2, Gaia TGAS, and UCAC5 catalog, respectively, at the epoch of each plate. In all the panels, the size of an image pixel is shown as a box, Gaussian fits are plotted over the histograms, and the Gaussian standard deviations are given.

plot for the triple-exposure plates is shown in Fig. 21. The photometric accuracy is $\sim 0.24 \mathrm{mag}$ for the triple-exposure plates.

\subsection{Triple-exposure images}

The results of the astrometric fit for the triple-exposure plates are in Tables A.1 and 3. The accuracy of astrometry is better than that of the single-exposure plates. This is expected because the stellar positions are calculated as a mean value of three images of a star. This also means that the astrometric accuracy of singleexposure plates is limited by the accuracy of the measured pixel positions of stars on a plate.

Figure 22 shows for each of the three stars within a triplet the distance of the star from the center of the triplet as a function of the total flux of the triplet. The plot is for plate \#854, including all the stars detected by our automatic procedure plus three manually selected, highly blended triplets not detected by our procedure (the three brightest stars). The star images at one of the corners of the asterisms (circles in Fig. 22) are located further away from the center than the star images at the other two corners. The reason for this is that stars in triplets are not at the same distance from the center of the mass of a triplet. The Kostinsky effect (increase of distance of star images in the triplet relative to each other as a function of stellar flux) is clearly detected. The functional form of the Kostinsky effect is similar to that found by Dick et al. (1993) and Ortiz-Gil et al. (1998); above a certain flux threshold, the relative distances of the stars in a triplet increase rapidly. In addition, our data show that for low fluxes the distances slightly decrease as a function of flux. We do not try to correct for this effect because the $x / y$ pixel coordinates of each triplet, the center of mass of a triplet, is calculated as a mean 

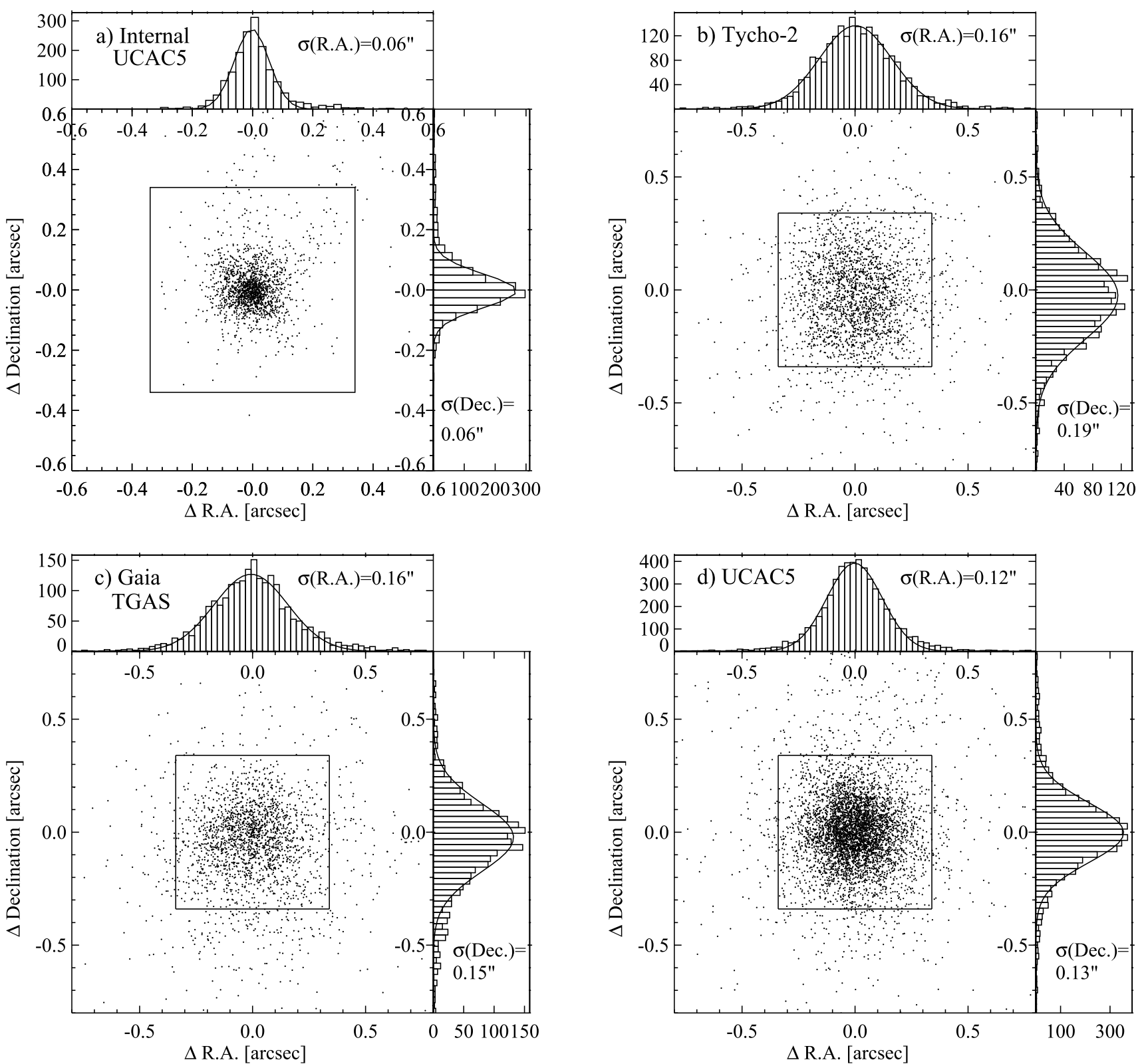

Fig. 17. Astrometric results for the triple-exposure plates. Panel $a$ : the differences between the coordinates of the UCAC5 stars in the overlapping images at the epoch of each plate. Panels $b-d$ : the differences between the coordinates of all the stars in the plates and the coordinates of the corresponding stars in the Tycho-2, Gaia TGAS, and UCAC5 catalog, respectively, at the epoch of each plate. In all the panels, the size of an image pixel is shown as a box, Gaussian fits are plotted over the histograms, and the Gaussian standard deviations are given.

value of the $x / y$ coordinates, which is expected to stay stationary even if the star images in the triplet move relative to the center of mass.

\section{Comparison with other CduC catalogs}

Rapaport et al. (2006) have analyzed 512 triple-exposure CduC plates, scanned with the Cambridge Automatic Plate Measuring (APM) machine. They give a value of $\sigma(\alpha \cos (\delta)) \simeq \sigma(\delta) \simeq$ $0.16^{\prime \prime}$ for the precision of a coordinate of a single image of a star near the center of a plate. Near the edges of a plate the precisions are $\sigma(\alpha \cos (\delta))=0.17^{\prime \prime}$ and $\sigma(\delta)=0.20^{\prime \prime}$. When considering the star triplets, the position uncertainty of the star of the triplet is lower by $\sqrt{3}$, and consequently they give an anticipated value of $\sim 0.11^{\prime \prime}$ for the astrometric standard errors.
To compare the positional accuracy obtained by Vicente et al. (2007, 2010) with our results, we quote their values for "reference star residuals of $\mathrm{CduC}$ catalog based on a comparison with the Tycho-2 positions at the epoch of the plates", with standard deviations of $\sigma(\alpha \cos (\delta))=0.22^{\prime \prime}$ and $\sigma(\delta)=0.24^{\prime \prime}$ (Fig. 8 of Vicente et al. 2010), including both single- and triple-exposure plates.

A single triple-exposure plate was scanned with a microdensitometer by Ortiz-Gil et al. (1998). They obtained astrometric accuracy of $\sigma(\Delta x)=0.16^{\prime \prime}$ and $\sigma(\Delta y)=0.13^{\prime \prime}$ when fitting the whole plate with ten reference stars.

The residuals in our survey, $\sigma(\alpha \cos (\delta))=0.17^{\prime \prime}$ and $\sigma(\delta)=$ $0.18^{\prime \prime}$ for the single-exposure plates in the case of Tycho-2 reference stars, are about equal to or lower than the residuals in the above-mentioned surveys. Our residuals for the triple-exposure plates, $\sigma(\alpha \cos (\delta))=0.16^{\prime \prime}$ and $\sigma(\delta)=0.19^{\prime \prime}$ for the Tycho- 2 


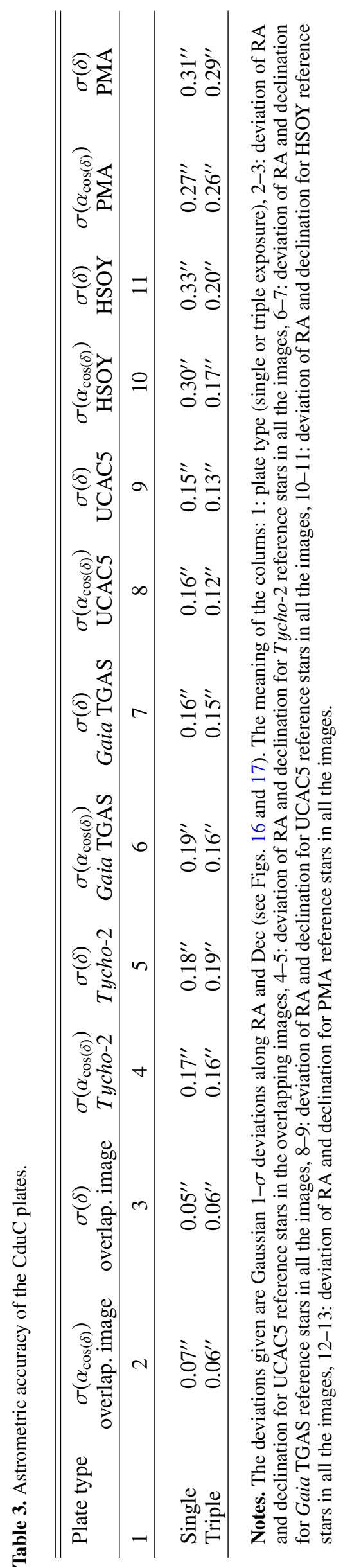

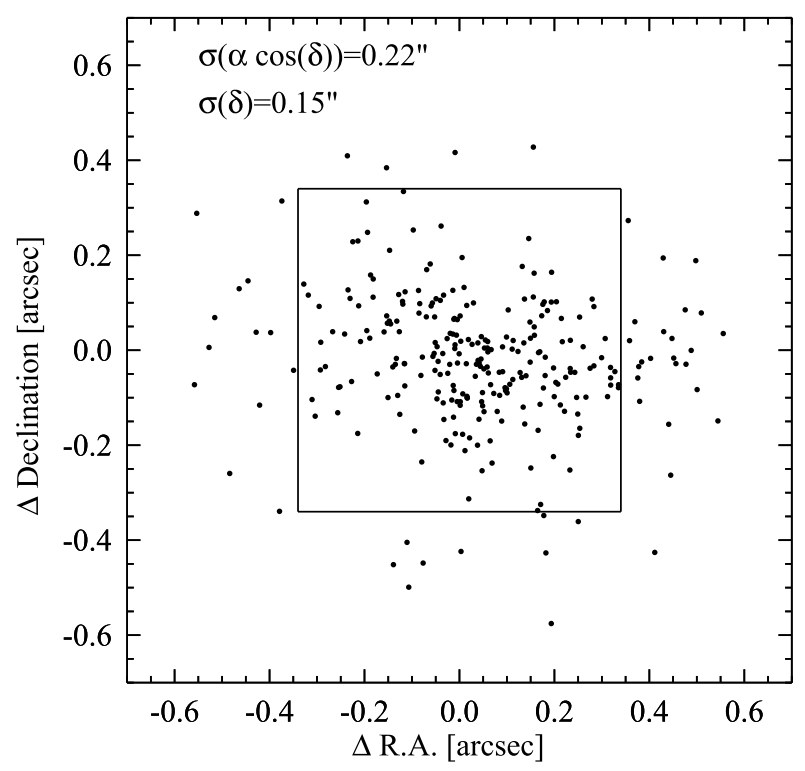

Fig. 18. Difference of the coordinates of the common stars in the partly overlapping plates \#892 and \#896. The size of an image pixel is shown as a box, and standard deviations are given.

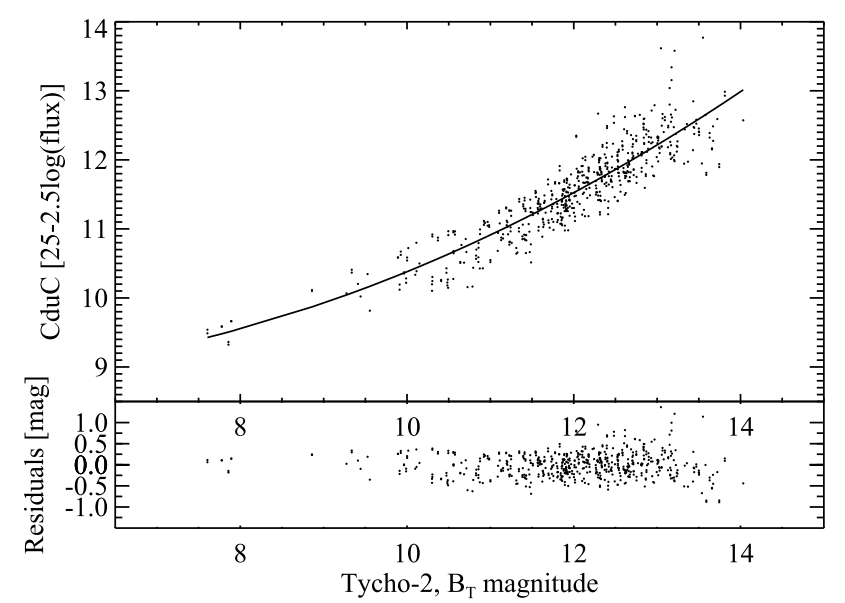

Fig. 19. Example of magnitude calibration. The upper panel shows the logarithm of the total flux of a star on a CduC plate as a function of the Tycho- $2 B_{T}$ magnitude. The lower panel shows the residuals after the overplotted second-order fit has been subtracted from the CduC magnitudes. This example is for plate \#841.

reference stars, are somewhat larger than those of Rapaport et al. (2006), but similar to those of Ortiz-Gil et al. (1998). It is important to note that the analyzed CduC plates were obtained with different physical telescopes at different observatories. Therefore, astrometric precisions not only stem from the analysis work.

Photographic magnitudes of stars on CduC plates have been obtained by Ortiz-Gil et al. (1998) with an accuracy of $0.09 \mathrm{mag}$, by Lamareille et al. (2003) with an accuracy of $\sim 0.2-0.4 \mathrm{mag}$, and by Rapaport et al. (2006) with an accuracy of 0.6 mag. We obtain similar photometric accuracy, 0.28 and 0.24 mag for single- and triple-exposure plates, respectively.

\section{Discussion}

Each plate has been digitized in two ways; (i) two overlapping images excluding the borders of the plates, and (ii) 


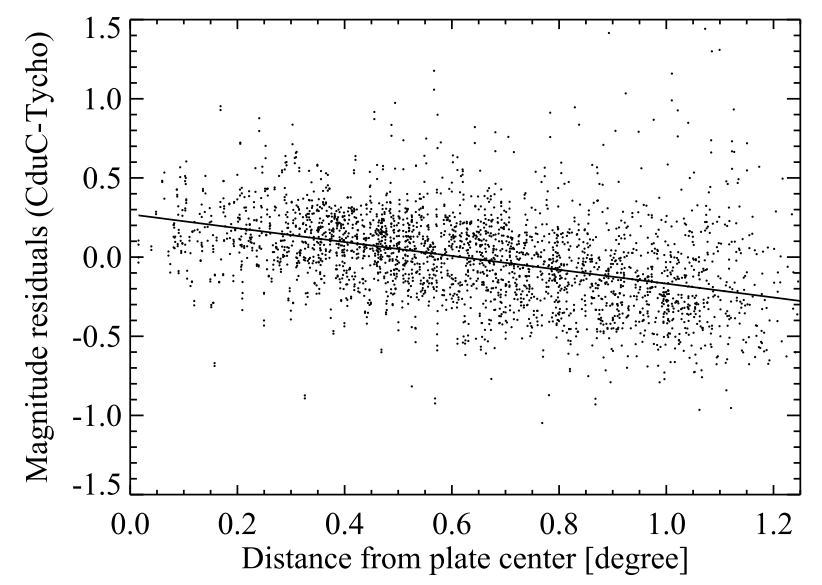

Fig. 20. Magnitude residuals (CduC magnitudes minus Tycho- $2 B_{T}$ magnitudes) as a function of the distance of the star from the center of the plate, for the single-exposure plates. A fit of a straight line to the data is shown.

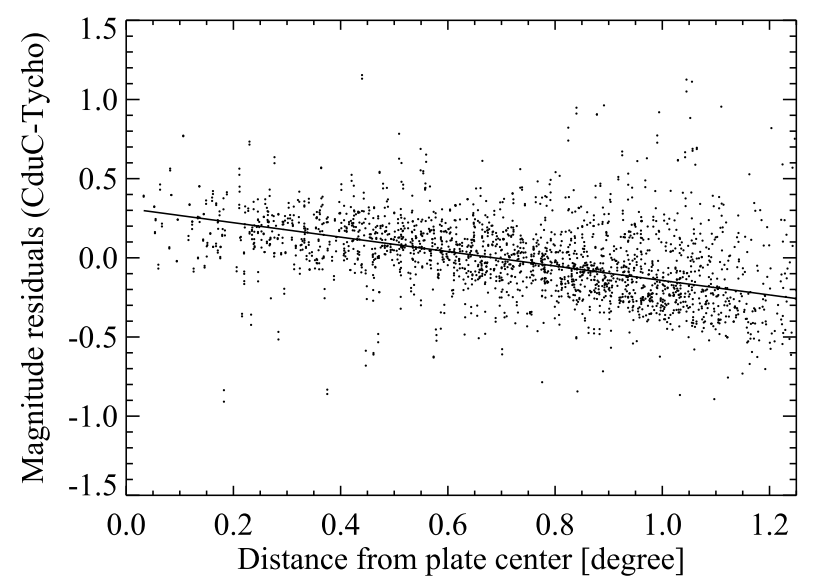

Fig. 21. Magnitude residuals (CduC magnitudes minus Tycho-2 $B_{T}$ magnitudes) as a function of the distance of the star from the center of the plate, for the triple-exposure plates. A fit of a straight line to the data is shown.

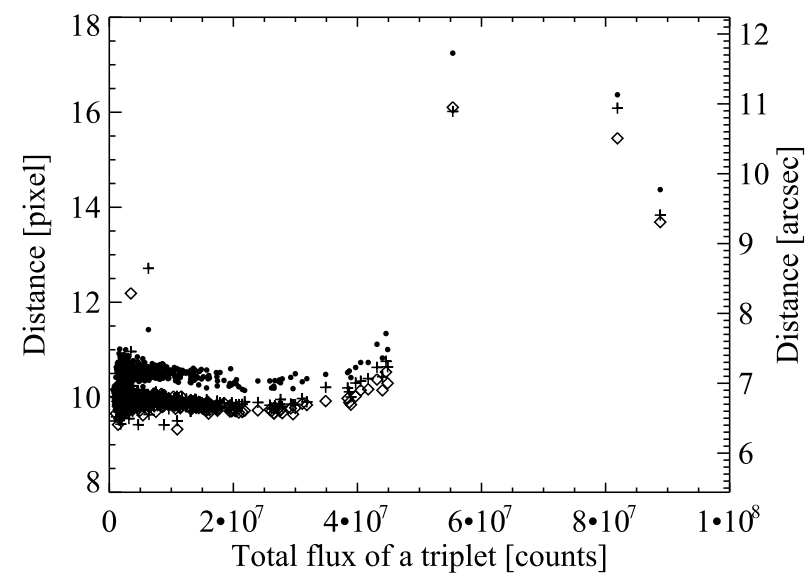

Fig. 22. Adjacency effect on the triple-exposure plate \#854. For each star image of a triplet, forming a triangle, the distance of the star from the center of mass of the triangle is plotted as a function of the total flux of the triplet. The stars at the different corners of the triangle are plotted with a different symbol (plus sign, square, circle).

four overlapping images covering the whole plate. The former method would be adequate to measure all the stars because the

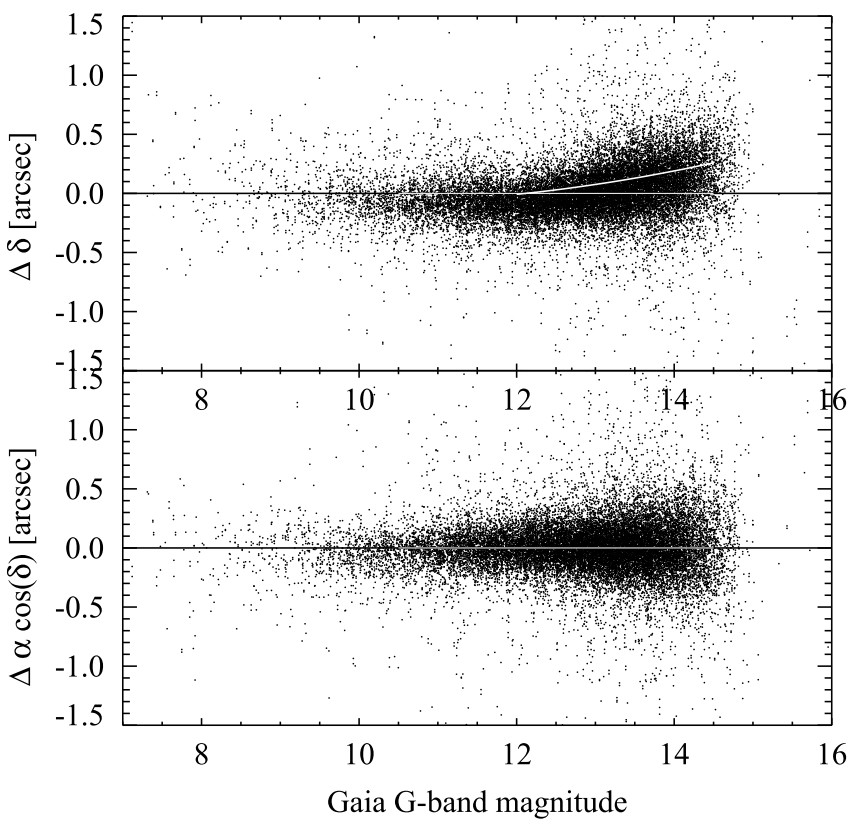

Fig. 23. Difference between the fitted and cataloged coordinates of all the UCAC5 stars in our single-exposure plates as a function of the Gaia $G$-band magnitude after we have modified the values of the declination proper motions given in the UCAC5 catalog. The amount of the additive

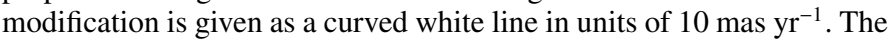
lower panel is for RA, the upper panel is for declination.

CduC plates are overlapping so that no star is lost. Furthermore, the former method gives astrometric residuals that are lower by $\sim 0.04 "$. However, we prefer the latter method for the following reasons: (i) The difference in astrometry is most probably caused by magnitude equation, which produces larger residuals at the borders of the plates (see Fig. 8). We can correct for this effect after we have digitized more plates. And (ii) If we exclude the borders of the plates, we lose photometric information on the stars in the excluded regions.

The uncertainty of the coordinates of the Gaia TGAS stars, at the epoch of the plates, is about two times lower than the uncertainty of the coordinates of the Tycho-2 stars (Fig. 3). However, we find that the accuracy of astrometry is similar for Gaia TGAS and Tycho-2 reference stars, even after correcting for the bias in Gaia TGAS data (Fig. 9). We give three explanations for the similarity:

(i) It is explained by underestimated Gaia TGAS proper motion uncertainties and/or overestimated Tycho-2 proper motion uncertainties, or by an unknown distortion in the Gaia TGAS data.

(ii) The pixel positions of stars on the CduC plates, measured by us, are biased in the same way as the coordinates of the Tycho- 2 reference stars, at the epoch of the plates. This biasing is probably caused by the large stellar profiles on photographic plates. Within a stellar profile, there may be dim stars that move the photocenter of the star on a plate away from the true position of the star. Thus, the stellar positions measured manually about 100 years ago, and digitally by us, are biased in the same way. On the other hand, the proper motions given in the Tycho- 2 catalog largely depend on the stellar positions from the Astrographic catalog. Thus, the stellar positions in the Helsinki section of the Astrographic catalog survey were used to derive the proper motions given in the Tycho-2 catalog. As a result, the positions of the stars calculated by using the non-biased proper motions given in the Gaia TGAS 
catalog do not give as good astrometry as would be expected based solely on the uncertainties of the proper motion values. On the other hand, we obtain the best astrometry with the UCAC5 reference star catalog, which is based on higher resolution ground-based CCD observations (and Gaia DR1 data). This suggests that the large stellar profiles on photographic plates are not the reason for the similar astrometric accuracy between Gaia TGAS and Tycho-2 data.

(iii) The intrinsic accuracy of the plates is worse or about the same as the accuracy of the coordinates of the Tycho- 2 reference stars at the epoch of the plates. Therefore, using more accurate Gaia TGAS reference stars does not improve astrometry. This explanation is not supported by the fact that, (i) the astrometric residuals become smaller when using only those reference stars for which the coordinate accuracy is limited (Table A.1), and (ii) astrometric accuracy is better when using the UCAC5 reference stars instead of the Gaia TGAS or Tycho-2 stars.

To test whether the bias in the astrometric residuals of the Gaia TGAS (Fig. 9) and HSOY (Fig. 13) data can be explained by biased proper motion values, we add a magnitude-dependent value to the declination proper motion values given in the (presumably unbiased) UCAC5 catalog. The functional form of the modification, shown in Fig. 23, is made similar to the bias seen in the astrometric residuals of the HSOY data (Fig. 13). The modification is limited to stars with Gaia $G$-band magnitude greater than

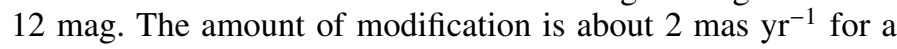
star with a Gaia $G$-band magnitude of 14 mag. We compute the coordinates of the UCAC5 stars at the epoch of the plates using the modified proper motions, and then we make astrometric fits. The residuals between the fitted and cataloged coordinates as a function of magnitude are shown in Fig. 23. The residuals along declination are biased in a way which resembles the bias in the proper motion values. The bias of the astrometric residuals at magnitude 14 is about 0.15 arcsec, which, after dividing with

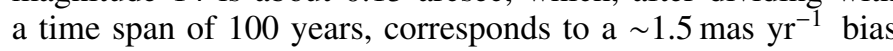
in proper motion, close to the value of the modification, $\sim 2$ mas $\mathrm{yr}^{-1}$. We thus conclude that, (i) our data analysis can detect a magnitude-dependent bias of proper motion values and give a correct value for the bias, and (ii) the bias in the astrometric residuals of the Gaia TGAS and HSOY data can be explained by their biased proper motion values.

Our conclusion on the biased Gaia TGAS proper motions is supported by Fedorov et al. (2018). They analyze the proper motions given in the Gaia TGAS, HSOY, UCAC5, and PMA catalogs under the assumption that systematic differences between the proper motions are caused by a mutual rigid-body rotation of the reference frames of the catalogs. They find that the published proper motions of the Tycho-2 stars in the Gaia TGAS catalog, derived with AGIS (Astrometric Global Iterative Solution), have a dependence on stellar magnitude. When deriving the proper motions in a classical way (the difference of the positions of HIPPARCOS/Tycho-2 - Gaia at the corresponding epochs divided by the span between the epochs), Fedorov et al. (2018) find that the published and classical proper motions of HIPPARCOS stars in the Gaia TGAS catalog are similar, while for Tycho-2 stars they are different. The dependence of the proper motions of the Tycho-2 stars on stellar magnitude is eliminated when the published Gaia TGAS proper motions are replaced with the classical ones.

The trend seen for magnitude residuals as a function of distance from the plate center in Figs. 20 and 21 is contrary to what is expected for vignetting by the telescope, which attenuates the stellar brightness outward from plate center. The trend is probably caused by the saturation effects of the emulsion. The stellar profiles in the plates are larger, and thus less saturated, at larger distances from the plate center. Therefore, we recover more of the original stellar flux at larger distances from the plate center. The difference between the $\mathrm{CduC}$ and Tycho magnitudes is zero at about half the distance from plate center to edge $\left(0.6^{\circ}\right)$ because the least squares fit of the magnitude calibration (Fig. 19) does not use information about the pixel coordinates of the stars.

The Gaia TGAS catalog has about two million sources. The upcoming (April 2018) Gaia DR2 is anticipated to have fiveparameter astrometric solutions for $>10^{9}$ sources. Then, (i) the accuracy of the astrometry is not limited by the accuracy of the proper motion values of the reference stars, (ii) we can study the effects of the possible close companion stars that are within the large stellar profiles of the CduC stars, and (iii) Gaia DR2 will contain photometry not only in the $G$-band, but also in the blue and red colors potentially allowing improvements in the CduC photometry calibration.

\section{Conclusions}

Six single-exposure and four triple-exposure $\mathrm{CduC}$ plates have been digitized with a digital camera and a macro lens, with a resolution of $\sim 0.7^{\prime \prime}$ per pixel. The astrometry for the images has been derived by using stars in the Tycho-2, Tycho-Gaia Astrometric Solution (Gaia TGAS), USNO CCD Astrograph Catalog (UCAC5), Hot Stuff for One Year (HSOY), and PMA catalogs as reference stars.

The best astrometric accuracy is obtained with the UCAC5 reference stars. The internal astrometric accuracy, that is, the deviation of the coordinates of the stars detected within overlapping images of a single plate, is $\sim 0.06^{\prime \prime}$. The external astrometric accuracy, that is, the deviation of the coordinates relative to the UCAC5 reference stars at the epoch of each plate, is $\sim 0.16^{\prime \prime}$ and $\sim 0.13^{\prime \prime}$ for the single- and triple-exposure plates, respectively. The plate-to-plate astrometric accuracy, that is, the deviation of the coordinates of the stars detected within two overlapping plates, is $\sim 0.21^{\prime \prime}$ along RA and $\sim 0.15^{\prime \prime}$ along declination. The photometric accuracy is $\sim 0.28 \mathrm{mag}$ and $\sim 0.24 \mathrm{mag}$ for the single- and triple-exposure plates, respectively.

The astrometric residuals of the UCAC5 and PMA stars at the epoch of the plates, and thus their proper motion values, are free of a magnitude-dependent bias. However, we believe that the RA proper motions in the Gaia TGAS catalog and the Declination proper motions in the HSOY catalog have a magnitudedependent bias. The magnitude of the bias is about 2 mas $\mathrm{yr}^{-1}$ at maximum.

There is a factor of about two difference in the astrometric quality between different plates, probably reflecting the intrinsic quality of emulsion on the plates. The astrometric precision is limited both by the precision of the stellar pixel positions measured on the plates and by the precision of the proper motion values of the reference stars.

Currently, we detect magnitude equation in our plates, but the number of the plates analyzed in our study is too low to properly determine a correction for magnitude equation. In the future the astrometric accuracy of our CduC plates will be improved because we will use data from the Gaia DR2 catalog, and we can make a correction for magnitude equation after we have digitized more plates.

Our current method to determine and remove the distortions caused by our instrumental setup is restricted by limitations in hardware and software, and thus not realized in this survey. On 


\section{K. Lehtinen et al.: Carte du Ciel and Gaia}

the other hand, removal of distortions caused by the instrumental setup would be justified only if the mechanical setup was absolutely non-time variable, which is not the case for our current setup. The astrometric accuracy obtained by us is equal or better than the accuracy of the other digitizing projects of the $\mathrm{CduC}$ plates.

We believe that a digital camera is a better instrument than a scanner to digitize the relatively small CduC plates. In the case of the camera Canon EOS 5Ds, it is sufficient to take four images of a CduC plate to achieve a good enough resolution.

Acknowledgements. We thank the late T. Markkanen who initiated this project, and whose wide knowledge of the Carte du Ciel survey was essential for this project. We would like to thank the anonymous referee for helpful comments. We thank Hervé Bouy and Emmanuel Bertin for assistance with the usage of Astromatic software. This work has made use of data from the European Space Agency (ESA) mission Gaia (https://www . cosmos.esa.int/Gaia), processed by the Gaia Data Processing and Analysis Consortium (DPAC, https://www. cosmos.esa.int/web/Gaia/dpac/ consortium). Funding for the DPAC has been provided by national institutions, in particular the institutions participating in the Gaia Multilateral Agreement.

\section{References}

Akhmetov, V. S., Fedorov, P. N., Velichko, A. B., \& Shulga, V. M. 2017, VizieR Online Data Catalog: I/341

Altmann, M., Roeser, S., Demleitner, M., Bastian, U., \& Schilbach, E. 2017, A\&A, 600, L4

Bertin, E. 2006, ASP Conf. Ser., 351, 112

Bertin, E., \& Arnouts, S. 1996, A\&AS, 117, 393
Bertin, E., Mellier, Y., Radovich, M., et al. 2002, ASP Conf. Ser., 281, 228 Bustos Fierro, I. H., \& Calderón, J. H. 2003, Rev. Mex. Astron. Astrofis., 39, 303 Dick, W. R., Tucholke, H.-J., Brosche, P., et al. 1993, A\&A, 279, 267 Donner, A. S. 1894, in Acta Soc. Scient. Fenn, Vol. Tome XX, No. 8 (Societas Scientiarum Fennica), 68

Donner, A. S. 1902, Öfversigt af Finska vetenskaps-societetens förhandlingar, XLIV, 8

Donner, A. S. 1929, in Catalogue photographique du ciel, Tome I, Fasc. 1 (University of Helsinki), 162

Donner, A. S., \& Furuhjelm, R. 1908, Catalogue photographique du ciel: (Helsingfors, Impr. societe anonyme SANA), 19

ESA, 1997, ESA SP, 1200

Fedorov, P. N., Akhmetov, V. S., \& Velichko, A. B. 2018, MNRAS, 476, 2743

Gaia Collaboration (Brown, A. G. A., et al.) 2016a, A\&A, 595, A2

Gaia Collaboration (Prusti, T., et al.) 2016b, A\&A, 595, A1

Geffert, M., Bonnefond, P., Maintz, G., \& Guibert, J. 1996, A\&AS, 118, 277

Girard, T. M., Platais, I., Kozhurina-Platais, V., van Altena, W. F., \& López, C. E. 1998, AJ, 115, 855

Høg, E., Fabricius, C., Makarov, V. V., et al. 2000, A\&A, 355, L27

Jones, D. 2000, Astronomy and Geophysics, 41, 16

Kostinskĭ, S. K. 1907, Mitteilungen der Nikolai-Hauptsternwarte zu Pulkowo, 2

Lamareille, F., Thiévin, J., Fournis, B., et al. 2003, A\&A, 402, 395

Lang, D., Hogg, D. W., Mierle, K., Blanton, M., \& Roweis, S. 2010, AJ, 139, 1782

Lindegren, L., Lammers, U., Bastian, U., et al. 2016, A\&A, 595, A4

Michalik, D., Lindegren, L., Hobbs, D., \& Butkevich, A. G. 2015, A\&A, 583, A68

Ortiz-Gil, A., Hiesgen, M., \& Brosche, P. 1998, A\&AS, 128, 621

Rapaport, M., Ducourant, C., Le Campion, J. F., et al. 2006, A\&A, 449, 435 Ross, F. E. 1921, ApJ, 53, 349

Shupe, D. L., Moshir, M., Li, J., et al. 2005, ASP Conf. Ser., 347, 491

Vicente, B., Abad, C., \& Garzón, F. 2007, A\&A, 471, 1077

Vicente, B., Abad, C., Garzón, F., \& Girard, T. M. 2010, A\&A, 509, A62

Zacharias, N., Finch, C., \& Frouard, J. 2017, AJ, 153, 166 


\section{Appendix A}

Table A.1. Results of astrometric solutions as given by SCAMP, both for internal and external calibration. dAXIS1 and dAXIS2 are along RA and declination, respectively.

\begin{tabular}{|c|c|c|c|c|c|c|c|c|c|c|}
\hline Plate & Catalog & $\begin{array}{c}\mathrm{dAXIS} 1_{\text {int }} \\
(\operatorname{arcsec})\end{array}$ & $\begin{array}{c}\mathrm{dAXIS} 2_{\text {int }} \\
(\operatorname{arcsec})\end{array}$ & $\chi_{\text {int }}^{2}$ & $\begin{array}{c}\mathrm{dAXIS} 1_{\text {ext }} \\
(\operatorname{arcsec})\end{array}$ & $\begin{array}{c}\text { dAXIS } 2_{\text {ext }} \\
(\operatorname{arcsec})\end{array}$ & $\chi_{\mathrm{ext}}^{2}$ & $\begin{array}{c}\text { Reference } \\
\text { accuracy }\end{array}$ & $\begin{array}{l}\text { Polyn. } \\
\text { degree }\end{array}$ & $\begin{array}{c}\text { Single/ } \\
\text { triple }\end{array}$ \\
\hline (1) & (2) & (3) & (4) & (5) & (6) & (7) & (8) & (9) & (10) & (11) \\
\hline \multirow[t]{9}{*}{841} & Gaia TGAS & 0.031 & 0.035 & 2.0 & 0.27 & 0.29 & 1.5 & & 3 & 1 \\
\hline & Gaia TGAS & 0.037 & 0.036 & 2.2 & 0.22 & 0.24 & 1.6 & $<0.15^{\prime \prime}$ & 3 & 1 \\
\hline & UCAC5 & 0.030 & 0.025 & 1.4 & 0.22 & 0.23 & 0.86 & & 3 & 1 \\
\hline & UCAC5 & 0.032 & 0.026 & 1.6 & 0.17 & 0.20 & 0.71 & $<0.20^{\prime \prime}$ & 3 & 1 \\
\hline & HSOY & 0.036 & 0.040 & 2.8 & 0.27 & 0.33 & 5.9 & & 3 & 1 \\
\hline & HSOY & 0.034 & 0.040 & 2.6 & 0.27 & 0.32 & 5.5 & $<0.27^{\prime \prime}$ & 3 & 1 \\
\hline & PMA & 0.030 & 0.026 & 1.5 & 0.33 & 0.36 & 0.79 & & 3 & 1 \\
\hline & PMA & 0.029 & 0.026 & 1.5 & 0.33 & 0.37 & 0.84 & $<0.32^{\prime \prime}$ & 3 & 1 \\
\hline & Tycho-2 & 0.028 & 0.028 & 1.5 & 0.24 & 0.25 & 0.85 & & 3 & 1 \\
\hline \multirow[t]{9}{*}{844} & Gaia TGAS & 0.032 & 0.031 & 2.0 & 0.24 & 0.19 & 0.94 & & 2 & 1 \\
\hline & Gaia TGAS & 0.036 & 0.034 & 2.5 & 0.21 & 0.18 & 1.1 & $<0.15^{\prime \prime}$ & 2 & 1 \\
\hline & UCAC5 & 0.028 & 0.022 & 1.3 & 0.17 & 0.16 & 0.44 & & 2 & 1 \\
\hline & UCAC5 & 0.028 & 0.022 & 1.3 & 0.15 & 0.14 & 0.38 & $<0.20^{\prime \prime}$ & 2 & 1 \\
\hline & HSOY & 0.030 & 0.026 & 1.6 & 0.27 & 0.26 & 2.2 & & 2 & 1 \\
\hline & HSOY & 0.030 & 0.026 & 1.6 & 0.27 & 0.26 & 2.1 & $<0.27^{\prime \prime}$ & 2 & 1 \\
\hline & PMA & 0.028 & 0.021 & 1.2 & 0.28 & 0.29 & 0.63 & & 2 & 1 \\
\hline & PMA & 0.028 & 0.020 & 1.2 & 0.28 & 0.28 & 0.63 & $<0.32^{\prime \prime}$ & 2 & 1 \\
\hline & Tycho-2 & 0.029 & 0.024 & 1.4 & 0.19 & 0.19 & 0.55 & & 2 & 1 \\
\hline \multirow[t]{9}{*}{883} & Gaia TGAS & 0.048 & 0.041 & 3.8 & 0.19 & 0.15 & 1.2 & & 2 & 1 \\
\hline & Gaia TGAS & 0.047 & 0.041 & 3.6 & 0.16 & 0.13 & 1.1 & $<0.15^{\prime \prime}$ & 2 & 1 \\
\hline & UCAC5 & 0.040 & 0.038 & 2.7 & 0.19 & 0.16 & 0.63 & & 2 & 1 \\
\hline & UCAC5 & 0.039 & 0.036 & 2.6 & 0.16 & 0.14 & 0.51 & $<0.20^{\prime \prime}$ & 2 & 1 \\
\hline & HSOY & 0.042 & 0.041 & 3.0 & 0.25 & 0.27 & 2.7 & & 2 & 1 \\
\hline & HSOY & 0.042 & 0.041 & 3.0 & 0.24 & 0.27 & 2.3 & $<0.27^{\prime \prime}$ & 2 & 1 \\
\hline & PMA & 0.039 & 0.035 & 2.4 & 0.26 & 0.28 & 0.55 & & 2 & 1 \\
\hline & PMA & 0.038 & 0.033 & 2.4 & 0.26 & 0.29 & 0.61 & $<0.32^{\prime \prime}$ & 2 & 1 \\
\hline & Tycho-2 & 0.042 & 0.039 & 3.0 & 0.19 & 0.17 & 0.53 & & 2 & 1 \\
\hline \multirow[t]{9}{*}{886} & Gaia TGAS & 0.033 & 0.027 & 1.8 & 0.25 & 0.19 & 1.4 & & 2 & 1 \\
\hline & Gaia TGAS & 0.035 & 0.027 & 1.9 & 0.21 & 0.16 & 1.0 & $<0.15^{\prime \prime}$ & 2 & 1 \\
\hline & UCAC5 & 0.027 & 0.022 & 1.1 & 0.18 & 0.17 & 0.56 & & 2 & 1 \\
\hline & UCAC5 & 0.027 & 0.022 & 1.2 & 0.14 & 0.14 & 0.40 & $<0.20^{\prime \prime}$ & 2 & 1 \\
\hline & HSOY & 0.027 & 0.022 & 1.2 & 0.40 & 0.45 & 3.6 & & 2 & 1 \\
\hline & HSOY & 0.028 & 0.022 & 1.2 & 0.39 & 0.44 & 3.6 & $<0.27^{\prime \prime}$ & 2 & 1 \\
\hline & PMA & 0.026 & 0.020 & 1.1 & 0.30 & 0.30 & 0.60 & & 2 & 1 \\
\hline & PMA & 0.026 & 0.021 & 1.1 & 0.30 & 0.31 & 0.66 & $<0.32^{\prime \prime}$ & 2 & 1 \\
\hline & Tycho-2 & 0.026 & 0.023 & 1.2 & 0.22 & 0.19 & 0.59 & & 2 & 1 \\
\hline \multirow[t]{9}{*}{890} & Gaia TGAS & 0.079 & 0.065 & 9.0 & 0.36 & 0.32 & 4.4 & & 3 & 1 \\
\hline & Gaia TGAS & 0.083 & 0.068 & 9.9 & 0.36 & 0.30 & 4.8 & $<0.15^{\prime \prime}$ & 3 & 1 \\
\hline & UCAC5 & 0.057 & 0.047 & 4.7 & 0.33 & 0.31 & 2.1 & & 3 & 1 \\
\hline & UCAC5 & 0.055 & 0.044 & 4.3 & 0.31 & 0.30 & 1.9 & $<0.20^{\prime \prime}$ & 3 & 1 \\
\hline & HSOY & 0.065 & 0.051 & 6.0 & 0.43 & 0.41 & 7.3 & & 3 & 1 \\
\hline & HSOY & 0.065 & 0.051 & 6.0 & 0.42 & 0.40 & 7.2 & $<0.27^{\prime \prime}$ & 3 & 1 \\
\hline & PMA & 0.067 & 0.064 & 8.0 & 0.40 & 0.39 & 1.0 & & 3 & 1 \\
\hline & PMA & 0.071 & 0.082 & 10.0 & 0.40 & 0.39 & 1.2 & $<0.32^{\prime \prime}$ & 3 & 1 \\
\hline & Tycho-2 & 0.052 & 0.039 & 3.6 & 0.37 & 0.34 & 1.7 & & 3 & 1 \\
\hline
\end{tabular}

Notes. The meaning of the colums: (1): plate number, (2): the catalog used for reference stars, (3) and (4): the internal RMS deviation of the astrometric fit along the axes of the images, (5): reduced $\chi^{2}$ goodness of the internal astrometric solution, (6) and (7): the external RMS deviation of the astrometric fit along the axes of the images, (8): reduced $\chi^{2}$ goodness of the external astrometric solution, (9): whether we use all the reference stars (empty) or only those stars which have positional uncertainty, at the epoch of the plates, less than the value given, (10): degree of the polynomial of the astrometric fit by SCAMP, (11): single- (1) or triple- (3) exposure plate. At the end of the table, the mean values of the parameters are given separately for the single and triple exposures. 
Table A.1. continued.

\begin{tabular}{|c|c|c|c|c|c|c|c|c|c|c|}
\hline Plate & Catalog & $\begin{array}{l}\mathrm{dAXIS} 1_{\text {int }} \\
(\operatorname{arcsec})\end{array}$ & $\begin{array}{c}\mathrm{dAXIS} 2_{\text {int }} \\
(\operatorname{arcsec})\end{array}$ & $\chi_{\text {int }}^{2}$ & $\begin{array}{l}\text { dAXIS1 } 1_{\text {ext }} \\
(\operatorname{arcsec})\end{array}$ & $\begin{array}{c}\mathrm{dAXIS} 2_{\text {ext }} \\
(\operatorname{arcsec})\end{array}$ & $\chi_{\text {ext }}^{2}$ & $\begin{array}{c}\text { Reference } \\
\text { accuracy }\end{array}$ & $\begin{array}{l}\text { Polyn. } \\
\text { degree }\end{array}$ & $\begin{array}{c}\text { Single/ } \\
\text { triple }\end{array}$ \\
\hline \multirow[t]{9}{*}{892} & Gaia TGAS & 0.037 & 0.032 & 2.2 & 0.23 & 0.18 & 1.3 & & 2 & 1 \\
\hline & Gaia TGAS & 0.039 & 0.032 & 2.4 & 0.22 & 0.17 & 1.5 & $<0.15^{\prime \prime}$ & 2 & 1 \\
\hline & UCAC5 & 0.035 & 0.025 & 1.8 & 0.17 & 0.15 & 0.64 & & 2 & 1 \\
\hline & UCAC5 & 0.032 & 0.024 & 1.6 & 0.16 & 0.14 & 0.56 & $<0.20^{\prime \prime}$ & 2 & 1 \\
\hline & HSOY & 0.044 & 0.031 & 2.7 & 0.28 & 0.27 & 4.3 & & 2 & 1 \\
\hline & HSOY & 0.043 & 0.030 & 2.6 & 0.28 & 0.26 & 4.2 & $<0.27^{\prime \prime}$ & 2 & 1 \\
\hline & PMA & 0.026 & 0.024 & 1.2 & 0.30 & 0.30 & 0.70 & & 2 & 1 \\
\hline & PMA & 0.027 & 0.025 & 1.3 & 0.29 & 0.29 & 0.71 & $<0.32^{\prime \prime}$ & 2 & 1 \\
\hline & Tycho-2 & 0.033 & 0.027 & 1.8 & 0.23 & 0.21 & 0.77 & & 2 & 1 \\
\hline \multirow[t]{9}{*}{854} & Gaia TGAS & 0.019 & 0.014 & 1.2 & 0.20 & 0.14 & 0.58 & & 2 & 3 \\
\hline & Gaia TGAS & 0.020 & 0.016 & 1.2 & 0.17 & 0.13 & 0.63 & $<0.15^{\prime \prime}$ & 2 & 3 \\
\hline & UCAC5 & 0.024 & 0.023 & 1.0 & 0.15 & 0.14 & 0.40 & & 2 & 3 \\
\hline & UCAC5 & 0.024 & 0.022 & 1.0 & 0.14 & 0.13 & 0.35 & $<0.20^{\prime \prime}$ & 2 & 3 \\
\hline & HSOY & 0.025 & 0.025 & 1.2 & 0.22 & 0.23 & 1.5 & & 2 & 3 \\
\hline & HSOY & 0.026 & 0.026 & 1.2 & 0.22 & 0.23 & 1.5 & $<0.27^{\prime \prime}$ & 2 & 3 \\
\hline & PMA & 0.029 & 0.023 & 1.4 & 0.28 & 0.30 & 0.61 & & 2 & 3 \\
\hline & PMA & 0.024 & 0.024 & 1.1 & 0.26 & 0.28 & 0.55 & $<0.32^{\prime \prime}$ & 2 & 3 \\
\hline & Tycho-2 & 0.018 & 0.016 & 1.1 & 0.16 & 0.17 & 0.48 & & 2 & 3 \\
\hline \multirow[t]{9}{*}{887} & Gaia TGAS & 0.021 & 0.025 & 1.9 & 0.19 & 0.21 & 1.5 & & 2 & 3 \\
\hline & Gaia TGAS & 0.022 & 0.026 & 2.0 & 0.18 & 0.20 & 1.6 & $<0.15^{\prime \prime}$ & 2 & 3 \\
\hline & UCAC5 & 0.028 & 0.026 & 0.9 & 0.17 & 0.20 & 0.67 & & 2 & 3 \\
\hline & UCAC5 & 0.028 & 0.026 & 0.9 & 0.15 & 0.17 & 0.55 & $<0.20^{\prime \prime}$ & 2 & 3 \\
\hline & HSOY & 0.035 & 0.034 & 1.9 & 0.22 & 0.31 & 3.3 & & 2 & 3 \\
\hline & HSOY & 0.034 & 0.035 & 1.9 & 0.22 & 0.31 & 3.3 & $<0.27^{\prime \prime}$ & 2 & 3 \\
\hline & PMA & 0.066 & 0.059 & 7.6 & 0.33 & 0.33 & 0.59 & & 2 & 3 \\
\hline & PMA & 0.058 & 0.112 & 20.0 & 0.31 & 0.36 & 0.80 & $<0.32^{\prime \prime}$ & 2 & 3 \\
\hline & Tycho-2 & 0.019 & 0.019 & 1.0 & 0.18 & 0.22 & 0.65 & & 2 & 3 \\
\hline \multirow[t]{9}{*}{894} & Gaia TGAS & 0.028 & 0.035 & 2.8 & 0.16 & 0.24 & 1.9 & & 2 & 3 \\
\hline & Gaia TGAS & 0.030 & 0.036 & 3.1 & 0.15 & 0.21 & 1.9 & $<0.15^{\prime \prime}$ & 2 & 3 \\
\hline & UCAC5 & 0.032 & 0.027 & 1.1 & 0.18 & 0.22 & 0.67 & & 2 & 3 \\
\hline & UCAC5 & 0.032 & 0.027 & 1.1 & 0.16 & 0.19 & 0.59 & $<0.20^{\prime \prime}$ & 2 & 3 \\
\hline & HSOY & 0.037 & 0.035 & 1.8 & 0.32 & 0.38 & 3.4 & & 2 & 3 \\
\hline & HSOY & 0.037 & 0.036 & 1.8 & 0.32 & 0.37 & 3.4 & $<0.27^{\prime \prime}$ & 2 & 3 \\
\hline & PMA & 0.031 & 0.026 & 0.98 & 0.29 & 0.34 & 0.65 & & 2 & 3 \\
\hline & PMA & 0.032 & 0.027 & 1.1 & 0.30 & 0.34 & 0.69 & $<0.32^{\prime \prime}$ & 2 & 3 \\
\hline & Tycho-2 & 0.022 & 0.018 & 1.2 & 0.16 & 0.21 & 0.61 & & 2 & 3 \\
\hline \multirow[t]{9}{*}{896} & Gaia TGAS & 0.020 & 0.019 & 0.75 & 0.16 & 0.13 & 0.5 & & 2 & 3 \\
\hline & Gaia TGAS & 0.020 & 0.019 & 0.79 & 0.13 & 0.12 & 0.5 & $<0.15^{\prime \prime}$ & 2 & 3 \\
\hline & UCAC5 & 0.023 & 0.021 & 0.71 & 0.16 & 0.15 & 0.55 & & 2 & 3 \\
\hline & UCAC5 & 0.023 & 0.021 & 0.73 & 0.14 & 0.13 & 0.46 & $<0.20^{\prime \prime}$ & 2 & 3 \\
\hline & HSOY & 0.026 & 0.022 & 0.84 & 0.21 & 0.20 & 1.5 & & 2 & 3 \\
\hline & HSOY & 0.026 & 0.022 & 0.84 & 0.21 & 0.20 & 1.5 & $<0.27^{\prime \prime}$ & 2 & 3 \\
\hline & PMA & 0.024 & 0.022 & 0.77 & 0.23 & 0.25 & 0.47 & & 2 & 3 \\
\hline & PMA & 0.025 & 0.022 & 0.78 & 0.24 & 0.24 & 0.49 & $<0.32^{\prime \prime}$ & 2 & 3 \\
\hline & Tycho-2 & 0.021 & 0.019 & 0.82 & 0.19 & 0.18 & 0.51 & & 2 & 3 \\
\hline Mean & Gaia TGAS & 0.043 & 0.039 & 3.5 & 0.26 & 0.22 & 1.8 & & & 1 \\
\hline \multirow[t]{8}{*}{ values } & Gaia TGAS & 0.046 & 0.040 & 3.8 & 0.23 & 0.20 & 1.9 & $<0.15^{\prime \prime}$ & & 1 \\
\hline & UCAC5 & 0.036 & 0.030 & 2.2 & 0.21 & 0.20 & 0.87 & & & 1 \\
\hline & UCAC5 & 0.035 & 0.029 & 2.1 & 0.18 & 0.18 & 0.74 & $<0.20^{\prime \prime}$ & & 1 \\
\hline & HSOY & 0.040 & 0.035 & 2.9 & 0.32 & 0.33 & 4.3 & & & 1 \\
\hline & HSOY & 0.040 & 0.035 & 2.8 & 0.31 & 0.33 & 4.1 & $<0.27^{\prime \prime}$ & & 1 \\
\hline & PMA & 0.036 & 0.032 & 2.6 & 0.31 & 0.32 & 0.71 & & & 1 \\
\hline & PMA & 0.036 & 0.035 & 2.9 & 0.31 & 0.32 & 0.78 & $<0.32^{\prime \prime}$ & & 1 \\
\hline & Tycho-2 & 0.035 & 0.030 & 2.1 & 0.24 & 0.23 & 0.83 & & & 1 \\
\hline
\end{tabular}


Table A.1. continued.

\begin{tabular}{|c|c|c|c|c|c|c|c|c|c|c|}
\hline Plate & Catalog & $\begin{array}{c}\mathrm{dAXIS1} 1_{\text {int }} \\
(\operatorname{arcsec})\end{array}$ & $\begin{array}{l}\text { dAXIS2 } 2_{\text {int }} \\
(\operatorname{arcsec})\end{array}$ & $\chi_{\mathrm{int}}^{2}$ & $\begin{array}{c}\mathrm{dAXIS} 1_{\text {ext }} \\
(\operatorname{arcsec})\end{array}$ & $\begin{array}{c}\mathrm{dAXIS} 2_{\mathrm{ext}} \\
(\operatorname{arcsec})\end{array}$ & $\chi_{\mathrm{ext}}^{2}$ & $\begin{array}{c}\text { Reference } \\
\text { accuracy }\end{array}$ & $\begin{array}{l}\text { Polyn. } \\
\text { degree }\end{array}$ & $\begin{array}{c}\text { Single/ } \\
\text { triple }\end{array}$ \\
\hline Mean & Gaia TGAS & 0.022 & 0.023 & 1.7 & 0.18 & 0.18 & 1.1 & & & 3 \\
\hline \multirow[t]{8}{*}{ values } & Gaia TGAS & 0.023 & 0.024 & 1.8 & 0.16 & 0.17 & 1.2 & $<0.15^{\prime \prime}$ & & 3 \\
\hline & UCAC5 & 0.027 & 0.024 & 0.93 & 0.16 & 0.18 & 0.57 & & & 3 \\
\hline & UCAC5 & 0.027 & 0.024 & 0.94 & 0.15 & 0.16 & 0.49 & $<0.20^{\prime \prime}$ & & 3 \\
\hline & HSOY & 0.031 & 0.029 & 1.4 & 0.24 & 0.28 & 2.4 & & & 3 \\
\hline & HSOY & 0.031 & 0.029 & 1.4 & 0.24 & 0.28 & 2.4 & $<0.27^{\prime \prime}$ & & 3 \\
\hline & PMA & 0.037 & 0.032 & 2.7 & 0.28 & 0.31 & 0.58 & & & 3 \\
\hline & PMA & 0.035 & 0.046 & 5.7 & 0.28 & 0.31 & 0.63 & $<0.32^{\prime \prime}$ & & 3 \\
\hline & Tycho-2 & 0.020 & 0.018 & 1.0 & 0.17 & 0.20 & 0.56 & & & 3 \\
\hline
\end{tabular}

